\title{
An Adaptive Modulation Scheme for Two-user Fading MAC with Quantized Fade State Feedback
}

\author{
Sudipta Kundu and B. Sundar Rajan \\ Dept. of ECE, IISc, Bangalore 560012, India, Email: \{sudiptak, bsrajan\} @ece.iisc.ernet.in
}

\begin{abstract}
With no CSI at the users, transmission over the twouser Gaussian Multiple Access Channel with fading and finite constellation at the input, is not efficient because error rates will be high when the channel conditions are poor. However, perfect CSI at the users is an unrealistic assumption in the wireless scenario, as it would involve massive feedback overheads. In this paper we propose a scheme which uses only quantized knowledge of CSI at the transmitters with the overhead being nominal. The users rotate their constellation without varying their transmit power to adapt to the existing channel conditions, in order to meet certain pre-determined minimum Euclidean distance requirement in the equivalent constellation at the destination. The optimal modulation scheme has been described for the case when both the users use symmetric $M$-PSK constellations at the input, where $M=2^{\lambda}, \lambda$ being a positive integer. The strategy has been illustrated by considering examples where both users use QPSK or 8-PSK signal sets at the input. It is shown that the proposed scheme has better throughput and error performance compared to the conventional non-adaptive scheme, at the cost of a feedback overhead of just $\left\lceil\log _{2}\left(\frac{M^{2}}{8}-\frac{M}{4}+2\right)\right\rceil+1$ bits, for the $M$-PSK case.
\end{abstract}

\section{INTRODUCTION}

A multiple access channel (MAC) consists of multiple users transmitting independent information to a common destination. There is no cooperation among the users. The capacity region for a discrete memoryless MAC is well known [1] [2]. For a two-user MAC with additive white Gaussian noise (AWGN) the capacity achieving input is the continuous Gaussian alphabet. The two-user Gaussian MAC with finite input constellations like $M$-QAM, $M$-PSK was studied in [3] [4]. It was shown that relative rotation between input constellations [3], or a constellation power allocation scheme [4] may be employed to maximize the constellation constrained (CC) capacity regions. Trellis based coding schemes were also suggested to achieve any rate pair within the CC capacity region.

In this paper, a two-user MAC with quasi-static fading is considered, as shown in Fig. 11 The two users transmit information to a common destination. The random variables $h_{1}$ and $h_{2}$ are the channel gains for User-1 and User-2 respectively and $h_{1}, h_{2} \sim \mathcal{C N}(0,1)$, where $\mathcal{C N}(0, s)$ denotes the circular symmetric complex Gaussian random variable with variance $s$. AWGN $z$ gets added to the received signal at the destination, $z \sim \mathcal{C N}\left(0, \sigma^{2}\right)$. User- $i$ transmits a symbol $x_{i}$ from a complex finite constellation $\mathcal{S}_{i}$ (like $M$-QAM or $M$-PSK) of unit average energy, i.e, $\mathbb{E}\left[\left|x_{i}\right|^{2}\right]=1$. Let $P$ be the average power constraint for each user. The received

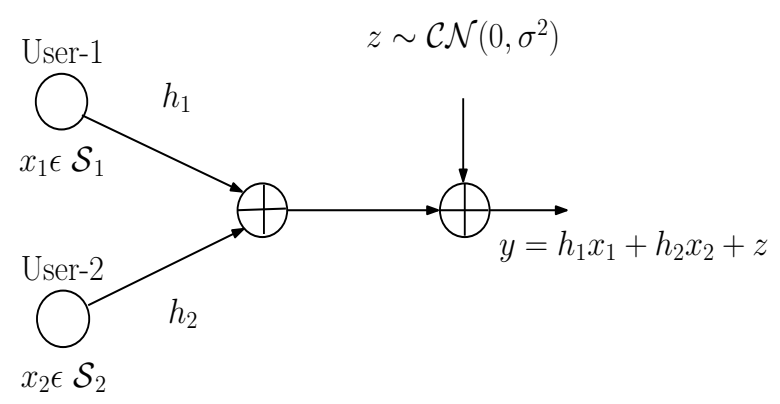

Fig. 1: Two-user fading MAC with Gaussian noise

signal at the destination is thus represented by

$$
y=\sqrt{P} h_{1} x_{1}+\sqrt{P} h_{2} x_{2}+z .
$$

We assume that perfect CSI i.e. the tuple $\left(h_{1}, h_{2}\right)$ is available only at the destination. At the destination the system can be viewed as a single user AWGN channel with the symbols drawn from a sum constellation

$$
\begin{aligned}
\mathcal{S}_{\text {sum }} & =\sqrt{P} h_{1} \mathcal{S}_{1}+\sqrt{P} h_{2} \mathcal{S}_{2} \\
& =\sqrt{P} h_{1}\left(\mathcal{S}_{1}+\frac{h_{2}}{h_{1}} \mathcal{S}_{2}\right) \\
& =\sqrt{P} h_{1} \underbrace{\left(\mathcal{S}_{1}+\gamma e^{j \theta} \mathcal{S}_{2}\right)}_{\mathcal{S}_{\text {eff }}},
\end{aligned}
$$

where $\gamma=\left|\frac{h_{2}}{h_{1}}\right|, \theta=\angle \frac{h_{2}}{h_{1}}$ and $\mathcal{S}_{\text {eff }}$ denotes the effective constellation.

Without loss of generality it can be assumed that $\gamma \geq 1$, as destination has knowledge of both $h_{1}$ and $h_{2}$ separately. If $\left|\frac{h_{2}}{h_{1}}\right|<1$, then at the destination the ratio can be simply reversed to compute $\frac{h_{1}}{h_{2}}$. Which one among the two ratios is calculated is made known to the users via a single bit of feedback. For the rest of the paper, we assume that the ratio $\frac{h_{2}}{h_{1}}$ is calculated at the destination. However, the results obtained still hold when the ratio calculated is $\frac{h_{1}}{h_{2}}$, by interchanging the roles of User-1 and User-2. For the rest of the paper a $M$ PSK constellation refers to a symmetric PSK signal set, with $M=2^{\lambda}, \lambda$ being a positive integer. The points in the $M$-PSK signal set are of the form $e^{\frac{(k-1) 2 \pi}{M}}$, where $1 \leq k \leq M$. We assume that $\mathcal{S}_{1}=\mathcal{S}_{2}=\mathcal{S}$, where $\mathcal{S}$ is an $M$-PSK constellation. We refer to the pair $(\gamma, \theta)$ to represent $\gamma e^{j \theta}$ and call it the fade state throughout the paper. We refer to the complex plane that represents $\gamma e^{j \theta}$ with $\gamma \geq 1$ as the $(\Gamma, \Theta)$ plane. 
Perfect channel state information (CSI) is available at the destination only, which quantizes the $(\Gamma, \Theta)$ plane into finite number of regions. The quantization obtained is similar to the one used for physical layer network coding in [5], which was subsequently derived analytically in [6]. This quantized knowledge of the fade state is made available to the users to adapt their modulation scheme via rotation of constellations to compensate for the possibly bad channel conditions. MAC with limited channel state information at transmitter (CSIT) has been studied from an information theoretic point of view in [7], [8]. In [9], it was shown that for a two-user discrete memoryless MAC with additional common message, finer CSIT results in increasing the capacity region. To the best of our knowledge, explicit modulation schemes with finite constellations and quantized fade state feedback has not been reported before.

The contributions and organization of this paper are as follows:

- A quantization of the $(\Gamma, \Theta)$ plane is derived, for the case when both users use $M$-PSK constellations at the input. We illustrate the quantization procedure by taking examples of the QPSK and 8-PSK case. (Section III-B)

- A modulation scheme is proposed for the users, which adapts according to the quantized feedback about the fade state that they receive from the destination, in order to satisfy a certain minimum distance guarantee $\delta$ in $\mathcal{S}_{\text {eff }}$ given in (1). The fade states which leads to violation of this minimum distance guarantee have been identified. Adaptation involves rotation of the constellation of one user relative to the other, without any change in transmit power, in order to effectively avoid these bad channel conditions. (Section III-A)

- The procedure to obtain the optimal angles for rotation is stated for the $M$-PSK case. The optimal rotation angles are calculated in closed form for the QPSK and 8-PSK case. (Section $\amalg$ II-B)

- An upper bound on $\delta$, i.e., the maximum value of the minimum distance in the effective constellation that can be guaranteed, is derived. (Section III-C)

- Simulation results are presented to show the extent to which the proposed strategy outperforms the conventional transmission scheme without adaptation. (Section IV)

\section{CHANNEL QUANTIZATION FOR $M$-PSK SIGNAL SETS}

In this section we obtain a quantization of the $(\Gamma, \Theta)$ plane into finite number of regions at the destination.

\section{A. Distance Distribution in the effective constellation}

Without loss of generality we assume that the average power constraint of each user is $P=1$. It is known that the error performance for an AWGN channel is determined by the Euclidean distance distribution of the input constellation. In our case, the distance distribution of $\mathcal{S}_{\text {sum }}$ decides the error performance at the destination. For any value of $(\gamma, \theta), d_{\left(s_{1}, s_{2}\right)_{\text {sum }} \leftrightarrow\left(s_{1}^{\prime}, s_{2}^{\prime}\right)_{\text {sum }}}^{2}$ denotes the distance between the two points $\left(s_{1}, s_{2}\right)_{\text {sum }}$ and $\left(s_{1}^{\prime}, s_{2}^{\prime}\right)_{\text {sum }}$, where $\left(s_{1}, s_{2}\right)_{\text {sum }}$, $\left(s_{1}^{\prime}, s_{2}^{\prime}\right)_{\text {sum }} \in \mathcal{S}_{\text {sum }}$ refer to the points $\sqrt{P} h_{1}\left(s_{1}+\gamma e^{j \theta} s_{2}\right)$ and $\sqrt{P} h_{1}\left(s_{1}^{\prime}+\gamma e^{j \theta} s_{2}^{\prime}\right)$ respectively with $s_{1}, s_{2}, s_{1}^{\prime}, s_{2}^{\prime} \in \mathcal{S}$. It is given by

$$
\begin{aligned}
d_{\left(s_{1}, s_{2}\right)_{\text {sum }} \leftrightarrow\left(s_{1}^{\prime}, s_{2}^{\prime}\right)_{\text {sum }}}^{2} & =P\left|h_{1}\right|^{2}\left|\left(s_{1}-s_{1}^{\prime}\right)+\gamma e^{j \theta}\left(s_{2}-s_{2}^{\prime}\right)\right|^{2} \\
& =P\left|h_{1}\right|^{2} d_{\left(s_{1}, s_{2}\right) \leftrightarrow\left(s_{1}^{\prime}, s_{2}^{\prime}\right)}^{2},
\end{aligned}
$$

where (2), $d_{\left(s_{1}, s_{2}\right) \leftrightarrow\left(s_{1}^{\prime}, s_{2}^{\prime}\right)}^{2}$ denotes the distance between the points $\left(s_{1}, s_{2}\right)$ and $\left(s_{1}^{\prime}, s_{2}^{\prime}\right)$, where $\left(s_{1}, s_{2}\right),\left(s_{1}^{\prime}, s_{2}^{\prime}\right)$ refers to the points $s_{1}+\gamma e^{j \theta} s_{2}$ and $s_{1}^{\prime}+\gamma e^{j \theta} s_{2}^{\prime}$ in $\mathcal{S}_{\text {eff. }}$. Since $P\left|h_{1}\right|^{2}$ simply scales the distances in $\mathcal{S}_{\text {eff }}$ we can focus only on

$$
d_{\left(s_{1}, s_{2}\right) \leftrightarrow\left(s_{1}^{\prime}, s_{2}^{\prime}\right)}^{2}=\left|\left(s_{1}-s_{1}^{\prime}\right)+\gamma e^{j \theta}\left(s_{2}-s_{2}^{\prime}\right)\right|^{2}
$$

as the quantity of interest.

It is clear from (3) that for certain values of $(\gamma, \theta)$ the distance between points $\left(s_{1}, s_{2}\right)$ and $\left(s_{1}^{\prime}, s_{2}^{\prime}\right)$ in $\mathcal{S}_{\text {eff }}$ reduces to zero, i.e. if

$$
\gamma e^{j \theta}=-\frac{\left(s_{1}-s_{1}^{\prime}\right)}{\left(s_{2}-s_{2}^{\prime}\right)}
$$

then $d_{\left(s_{1}, s_{2}\right) \leftrightarrow\left(s_{1}, s_{2} \prime\right)}^{2}=0$. These values of $(\gamma, \theta)$ are called the singular fade states [5], [6]. Singular fade states can also be defined as follows:

Definition 1: A fade state $(\gamma, \theta)$ is said to be a singular fade state if $\left|\mathcal{S}_{\text {eff }}\right|<M^{2}$.

Clearly, $\gamma e^{j \theta}=0$ is a singular fade state, for any arbitrary signal set $\mathcal{S}$. For any input constellation $\mathcal{S}$ the other non-zero singular fade states are obtained using (4). For a given input constellation $\mathcal{S}$, let $\mathcal{H}$ denote the set of all singular fade states.

Example 1: When $\mathcal{S}_{1}=\mathcal{S}_{2}=\mathcal{S}$, where $\mathcal{S}$ is a QPSK constellation, then the non-zero singular fade states are at

$$
\begin{aligned}
& \gamma=\sqrt{2}, \quad \theta=45^{\circ}, 135^{\circ}, 225^{\circ}, 315^{\circ} \\
& \gamma=1, \quad \theta=0^{\circ}, 90^{\circ}, 180^{\circ}, 270^{\circ} \\
& \gamma=\frac{1}{\sqrt{2}}, \theta=45^{\circ}, 135^{\circ}, 225^{\circ}, 315^{\circ}
\end{aligned}
$$

Since for an AWGN channel the error performance at the destination is dominated by the minimum distance of the input constellation, it is sufficient to study the minimum distance of $\mathcal{S}_{\text {eff. }}$. Also from Definition 1 minimum distance in $\mathcal{S}_{\text {eff }}$ reduces to zero at the singular fade states. The following lemma provides an upper bound on the minimum distance of the effective constellation $\mathcal{S}_{\text {eff }}$.

Lemma 1: When both the users use any arbitrary signal set $\mathcal{S}$ (which includes $M$-PSK, $M$-QAM) at the input, then for any fade state $(\gamma, \theta)$, the minimum distance $d_{\min }(\gamma, \theta)$ between any two points in $\mathcal{S}_{\text {eff }}$ is upper bounded by the minimum distance in the input constellation $d_{\min }(\mathcal{S})$.

Proof: From the definition of $d_{\min }^{2}(\gamma, \theta)$, we have,

$$
\begin{array}{r}
d_{\text {min }}^{2}(\gamma, \theta) \underset{\left.\left(s_{1}, s_{2}\right) \neq \min _{1}^{\prime}, s_{2}^{\prime}\right) \in \mathcal{S}^{2}}{ }\left|\left(s_{1}-s_{1}^{\prime}\right)+\gamma e^{j \theta}\left(s_{2}-s_{2}^{\prime}\right)\right|^{2} \\
\underset{\left(s_{1}, s_{2}\right) \neq\left(s_{1}^{\prime}, s_{2}^{\prime}\right) \in \mathcal{S}^{2}}{ }\left\{\left|s_{1}-s_{1}^{\prime}\right|^{2}+\gamma^{2}\left|s_{2}-s_{2}^{\prime}\right|^{2}\right\}
\end{array}
$$




$$
\begin{aligned}
& \text { Now } d_{\min }^{2}(\gamma, \theta) \leq \min _{s_{1} \neq s_{1}^{\prime} \in \mathcal{S}}\left|s_{1}-s_{1}^{\prime}\right|^{2}=d_{m i n}^{2}(\mathcal{S}) . \\
& \text { Also } d_{\min }^{2}(\gamma, \theta) \leq \min _{s_{2} \neq s_{2}^{\prime} \in \mathcal{S}} \gamma^{2}\left|s_{2}-s_{2}^{\prime}\right|^{2}=\gamma^{2} d_{\min }^{2}(\mathcal{S}) .
\end{aligned}
$$

From (5) and (6), and using the fact that $\gamma \geq 1$, we have

$$
d_{\text {min }}^{2}(\gamma, \theta) \leq \min \left\{d_{\text {min }}^{2}(\mathcal{S}), \gamma^{2} d_{\min }^{2}(\mathcal{S})\right\}=d_{\min }^{2}(\mathcal{S}) .
$$

In the following lemma, it is proved that in order to study the distance profile in $\mathcal{S}_{\text {eff }}$ it is sufficient to consider $\theta \in[0, \pi / M]$ when both users use $M$-PSK signal sets. Distance profiles for other values of $\theta$ can be obtained from $\theta \in[0, \pi / M]$. We use the term wedge $\left[\theta_{1}, \theta_{2}\right]$ to denote the region $\gamma \geq 1$ and $\theta \in\left[\theta_{1}, \theta_{2}\right]$ on the $(\Gamma, \Theta)$ plane. The lines $\theta=\theta_{1}$ and $\theta=\theta_{2}$ for $\gamma \geq 1$ and the arc $\gamma=1$ for $\theta \in\left[\theta_{1}, \theta_{2}\right]$ form the boundary of the wedge $\left[\theta_{1}, \theta_{2}\right]$.

Lemma 2: To study the distance profile in $\mathcal{S}_{\text {eff }}$ when both the users use $M$-PSK constellations, it is sufficient to consider the case $0 \leq \theta \leq \pi / M$. All other cases can be obtained from this.

Proof: The proof is in two steps. First we show that the distance profile is a repetitive structure with period $2 \pi / M$. Next, it is shown that within the wedge $[0,2 \pi / M]$ the distance profile is symmetric about the bisector of this wedge i.e., the $\theta=\pi / M$ line. We have from (1),

$$
\mathcal{S}_{\text {eff }}=\mathcal{S}+\gamma e^{j \theta} \mathcal{S} .
$$

For any arbitrary value of $\theta=\frac{k 2 \pi}{M}+\theta^{\prime}$ where $k \in \mathbb{Z}, 0 \leq$ $\theta^{\prime}<\frac{2 \pi}{M}$

$$
\begin{aligned}
\mathcal{S}_{\text {eff }} & =\mathcal{S}+\gamma e^{j \theta^{\prime}}\left(\mathcal{S} e^{j \frac{2 k \pi}{M}}\right) \\
& =\mathcal{S}+\gamma e^{j \theta^{\prime}} \mathcal{S} .
\end{aligned}
$$

The last equality follows from the fact that rotating a $M$ PSK constellation by an integral multiple of $2 \pi / M$ does not alter the distance profile of the constellation. Thus, whatever distance profiles for $\mathcal{S}_{\text {eff }}$ are obtained for the wedge $[0,2 \pi / M]$, it is exactly repeated for the remaining $M-1$ wedges to cover the entire range of $\theta$.

To show that the distance profiles are symmetric about $\theta=\pi / M$, we need to show that $\mathcal{S}+\gamma e^{j\left(\frac{\pi}{M}+\alpha\right)} \mathcal{S}$ and $\mathcal{S}+\gamma e^{j\left(\frac{\pi}{M}-\alpha\right)} \mathcal{S}$, where $0 \leq \alpha \leq \pi / M$, have the same distance profiles. We have

$$
\begin{aligned}
\mathcal{S}+\gamma e^{j\left(\frac{\pi}{M}-\alpha\right)} \mathcal{S} & =\mathcal{S}+\gamma e^{j\left(\frac{\pi}{M}-\alpha\right)}\left(\mathcal{S} e^{\frac{-2 \pi}{M}}\right) \\
& =\mathcal{S}+\gamma e^{-j\left(\frac{\pi}{M}+\alpha\right)} \mathcal{S} .
\end{aligned}
$$

The first equality is because $\mathcal{S}=\mathcal{S} e^{j k 2 \pi / M}$, i.e. rotating $\mathcal{S}$ by $2 k \pi / M$ gives the same constellation. Thus for $k=-1$, $\mathcal{S}=\mathcal{S} e^{\frac{-2 \pi}{M}}$. Also due to the symmetric nature of $M$-PSK constellation, the distance distribution of the sum constellation depends only on the relative angle of rotation between the input constellations. Thus $\mathcal{S}+\gamma e^{\beta} \mathcal{S}$ and $\mathcal{S}+\gamma e^{-\beta} \mathcal{S}$ have same distance profiles, for any $\beta \in[0, \pi]$. This together with (7) proves the second part of the lemma.
From Lemma2 it is clear that when both users use $M$-PSK signal sets, if $\left(\gamma^{\prime}, \theta^{\prime}\right)$ is a singular fade state, then there exists singular fade states at $\left(\gamma^{\prime}, \theta^{\prime}+p \frac{2 \pi}{M}\right)$, where $1 \leq p \leq M-1$ because distance distribution in $\mathcal{S}_{\text {eff }}$ is periodic with period $\frac{2 \pi}{M}$. The distance distribution of $\mathcal{S}_{\text {eff }}$ is the basis for channel quantization. Also from Lemma 2 , it suffices to obtain such a quantization only for the wedge $[0, \pi / M]$. This can then be reflected along the $\theta=\pi / M$ line, to give the quantization for the wedge $[0,2 \pi / M]$, which when repeated for the remaining $M-1$ wedges will cover the entire $(\Gamma, \Theta)$ plane.

\section{B. Channel Quantization for the M-PSK case}

In this subsection we propose a technique to obtain the quantization of the $(\Gamma, \Theta)$ plane, when both users use $M$ PSK signal sets. From Lemma 1, when both users use $M$-PSK signal sets at the input, the minimum distance in $\mathcal{S}_{\text {eff }}$ for any value of $(\gamma, \theta), d_{\min }(\gamma, \theta) \leq d_{\min }(\mathcal{S})=\sqrt{2\left[1-\cos \left(\frac{2 \pi}{M}\right)\right]}$. Now the following lemma gives the number of singular fade states in the wedge $[0, \pi / M]$.

Lemma 3: When both users use $M$-PSK signal sets at the input, the number of singular fade states in the wedge $[0, \pi / M]$, is given by $\frac{M^{2}}{8}-\frac{M}{4}+1$. Further, these singular fade states lie along the two lines $\theta=0$ and $\theta=\pi / M$.

Proof: From [6], the total number of singular fade states other than zero is $\frac{M^{3}}{4}-\frac{M^{2}}{2}+M$. Out of these, $M$ lie on the circle $\gamma=1$. It is also known from [6], that if $\gamma e^{j \theta}$ is a singular fade state, then $\frac{1}{\gamma} e^{-j \theta}$ is also a singular fade state. Thus, half of the total number of remaining singular fade states lie inside the circle $\gamma=1$ and the other half lies outside it. This along with the fact that singular fade states are periodic, implies the number of singular fade states for the wedge $[0, \pi / M]$, is given by

$$
\frac{1}{M}\left(\frac{\frac{M^{3}}{4}-\frac{M^{2}}{2}}{2}+M\right)=\frac{M^{2}}{8}-\frac{M}{4}+1 .
$$

Also from [6], it is clear that these fade states lie along $\theta=0$ and $\theta=\pi / M$ lines.

We denote this set of all singular fade states lying in the wedge $[0, \pi / M]$ by $\mathcal{H}_{W}$. Let $N_{W}=\left|\mathcal{H}_{W}\right|$.

Observe from (3), that the distance between two points in $\mathcal{S}_{\text {eff }}$ is a function of $\gamma$ and $\theta$. Let $\left|\triangle s_{j}\right|=\left|s_{j}-s_{j}^{\prime}\right|$ and $\phi_{j}=\angle\left(s_{j}-s_{j}^{\prime}\right)$ for $j=1,2$. Now from (3), the distance between the elements of the pair $\left\{\left(s_{1}, s_{2}\right),\left(s_{1}^{\prime}, s_{2}^{\prime}\right)\right\} \in \mathcal{S}_{\text {eff }}^{2}$ is

$d_{\left(s_{1}, s_{2}\right) \leftrightarrow\left(s_{1}^{\prime}, s_{2}^{\prime}\right)}^{2}=\left|\triangle s_{1}\right|^{2}+\gamma^{2}\left|\triangle s_{2}\right|^{2}+2 \gamma\left|\triangle s_{1} \triangle s_{2}\right| \cos \left(\theta+\phi_{2}-\phi_{1}\right)$.

Consider another other pair $\left\{\left(\hat{s}_{1}, \hat{s}_{2}\right),\left(\hat{s}_{1}^{\prime}, \hat{s}_{2}^{\prime}\right)\right\} \in \mathcal{S}_{\text {eff }}^{2}$ with $\hat{s}_{1}, \hat{s}_{1}^{\prime}, \hat{s}_{2}, \hat{s}_{2}^{\prime} \in \mathcal{S}$, and let $\left|\triangle \hat{s}_{j}\right|=\left|\hat{s}_{j}-\hat{s}_{j}^{\prime}\right|$ and $\hat{\phi}_{j}=\angle\left(\hat{s}_{j}-\right.$ $\left.\hat{s}_{j}^{\prime}\right)$ for $j=1,2$. If $\left|\triangle \hat{s}_{1}\right|=\left|\triangle s_{1}\right|,\left|\triangle \hat{s}_{2}\right|=\left|\triangle s_{2}\right|$ and $\hat{\phi}_{2}-\hat{\phi}_{1}=\phi_{2}-\phi_{1}$ or $\hat{\phi}_{2}-\hat{\phi}_{1}=\pi-\left(\phi_{2}-\phi_{1}\right)$, then from (8), $d_{\left(\hat{s}_{1}, \hat{s}_{2}\right) \leftrightarrow\left(\hat{s}_{1}^{\prime}, \hat{s}_{2}^{\prime}\right)}^{2}=d_{\left(s_{1}, s_{2}\right) \leftrightarrow\left(s_{1}^{\prime}, s_{2}^{\prime}\right)}^{2}$ for all values of $(\gamma, \theta)$, even though the value of this distance changes with $(\gamma, \theta)$.

Definition 2: A distance class denoted by $\mathcal{C}$, is a subset of $\mathcal{S}_{\text {eff }}^{2}$, which contains the pairs of the form $\left\{\left(s_{1}, s_{2}\right),\left(s_{1}^{\prime}, s_{2}^{\prime}\right)\right\}$, 


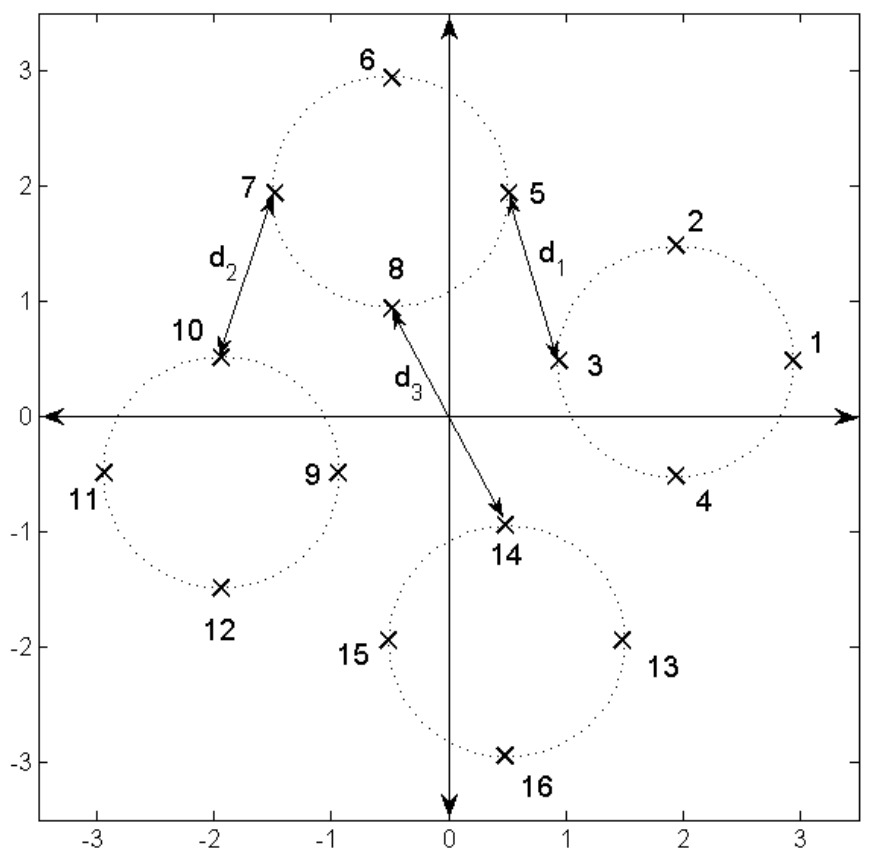

Fig. 2: $\mathcal{S}_{\text {eff }}$ constellation, when both users use QPSK signal sets, for $(\gamma, \theta)=\left(2,14^{\circ}\right)$. In the figure $d_{i}$ stands for the class distance function $d_{\mathcal{C}_{k_{i}}}(\gamma, \theta)$.

$\left(s_{1}, s_{2}\right) \neq\left(s_{1}^{\prime}, s_{2}^{\prime}\right)$ where $\left(s_{1}, s_{2}\right)$ and $\left(s_{1}^{\prime}, s_{2}^{\prime}\right)$ denote the complex points in $\mathcal{S}_{\text {eff }}$, such that the distance between the two elements of a pair is same for all pairs in $\mathcal{C}$ and this property holds for all values of $\gamma$ and $\theta$, though the value of the distance depends on $(\gamma, \theta)$.

For a given input constellation $\mathcal{S}$, let $\overline{\mathcal{C}}$ denote the set of the all distance classes for it.

Definition 3: Associated with every distance class $\mathcal{C}$ is a function $d_{\mathcal{C}}(\gamma, \theta):(\Gamma, \Theta) \leftrightarrow \mathbb{R}$, called the class distance function, which gives the value of the distance between the two elements of a pair in $\mathcal{C}$ for any $(\gamma, \theta)$.

Definition 4: For a given fade state $(\gamma, \theta)$, the function $d_{\gamma, \theta}(\mathcal{C}): \overline{\mathcal{C}} \rightarrow \mathbb{R}$ gives the value of the distance between the two elements of a pair in $\mathcal{C}$, for any $\mathcal{C} \in \overline{\mathcal{C}}$. This is called the fade state distance function.

We use integer $m, 1 \leq m \leq M$ to represent the point $e^{j \frac{(m-1) 2 \pi}{M}}$ in $\mathcal{S}$ i.e. the $M$-PSK signal set. The integer $q=$ $m+M(n-1), 1 \leq q \leq M^{2}$ denotes the complex point in $\mathcal{S}_{\text {eff }}$ obtained by combining the points $m$ and $n$ of $\mathcal{S}$ i.e. it refers to the point $e^{j \frac{(m-1) 2 \pi}{M}}+\gamma e^{j \theta} e^{j \frac{(n-1) 2 \pi}{M}}$ in $\mathcal{S}_{\text {eff }}$. For each distance class $\mathcal{C}$, among all the pairs $(i, j) \in \mathcal{C}$ choose the one with the minimum value of $i+j$ to be the representative in $\mathcal{C}$. If more than one pair has the same value of $i+j$ choose the one with the lowest value of $i$ as the class representative. When the users $M$-PSK signal set at input, there are $\frac{M^{2}\left(M^{2}-1\right)}{2}$ pairwise distances in $\mathcal{S}_{\text {eff }}$. These pairwise distances are thus partitioned into distance classes.

Example 2: Fig. 2] shows $\mathcal{S}_{\text {eff }}$ when both users use QPSK constellations for $(\gamma, \theta)=\left(2,14^{\circ}\right)$. There are 120 pairwise distances and 20 distance classes. These are listed in Table [ along with the corresponding class distance functions, and the class representatives.

We define the set of all class distance function, $d_{\overline{\mathcal{C}}}(\gamma, \theta)$ and the set of all fade state distance functions $d_{\Gamma, \Theta}(\mathcal{C})$ as follows,

$$
\begin{aligned}
& d_{\overline{\mathcal{C}}}(\gamma, \theta)=\left\{d_{\mathcal{C}}(\gamma, \theta) \mid \mathcal{C} \in \overline{\mathcal{C}}\right\} \\
& d_{\Gamma, \Theta}(\mathcal{C})=\left\{d_{\gamma, \theta}(\mathcal{C}) \mid(\gamma, \theta) \in(\Gamma, \Theta) \text { plane }\right\} .
\end{aligned}
$$

From Definition 1, at a singular fade state the value of at least one of the class distance functions in $d_{\overline{\mathcal{C}}}(\gamma, \theta)$ will reduce to zero.

Lemma 4: Among the set of all class distance functions that reduce to zero at the singular fade state $\left(\gamma^{\prime}, \theta^{\prime}\right)$, there is a particular one which is the minimum among that set, for all values of $(\gamma, \theta) \neq\left(\gamma^{\prime}, \theta^{\prime}\right)$.

Proof: Let $L$ be the number of class distance functions that reduce to zero at the singular fade state $\left(\gamma^{\prime}, \theta^{\prime}\right)$. Denote these by $d_{\mathcal{C}_{i}}(\gamma, \theta), 1 \leq i \leq L$ and let $\left\{\left(s_{1, i}, s_{2, i}\right),\left(s_{1, i}^{\prime}, s_{2, i}^{\prime}\right)\right\}$ be the representative element for the distance class $\mathcal{C}_{i}$. From (3) and (4), we have

$$
\begin{aligned}
d_{\mathcal{C}_{i}}^{2}(\gamma, \theta) & =d_{\left(s_{1, i}, s_{2, i}\right) \leftrightarrow\left(s_{1, i}^{\prime}, s_{2, i}^{\prime}\right)}^{2} \\
& =\left|\left(s_{1, i}-s_{1, i}^{\prime}\right)+\gamma e^{j \theta}\left(s_{2, i}-s_{2, i}^{\prime}\right)\right|^{2} \\
& =\left|s_{2, i}-s_{2, i}^{\prime}\right|^{2}\left|\gamma e^{j \theta}+\frac{s_{1, i}-s_{1, i}^{\prime}}{s_{2, i}-s_{2, i}^{\prime}}\right|^{2} \\
& =\left|s_{2, i}-s_{2, i}^{\prime}\right|^{2}\left|\gamma e^{j \theta}-\gamma^{\prime} e^{j \theta^{\prime}}\right|^{2} .
\end{aligned}
$$

From (9), these $L$ class distance functions differ only in the constant coefficient $\left|s_{2, i}-s_{2, i}^{\prime}\right|^{2}$. From Definition 2, all these coefficients are different. Let

$$
l^{\prime}=\arg \min _{1 \leq i \leq L}\left|s_{2, i}-s_{2, i}^{\prime}\right|^{2} .
$$


TABLE I: Distance classes when both users use QPSK signal set.

\begin{tabular}{|c|l|l|l|}
\hline$k$ & $\mathcal{C}_{k}$ & $d_{\mathcal{C}_{k}}(\gamma, \theta)$ & Class representative \\
\hline 1 & $(1,2),(1,4),(2,3),(3,4),(5,6),(6,7),(7,8),(5,8)$, & $\sqrt{2}$ & $(1,2)$ \\
& $(9,1),(10,11),(11,12),(9,12),(13,14),(14,15),(15,16),(13,16)$ & & $(1,3)$ \\
\hline 2 & $(1,3),(2,4),(5,7),(6,8),(9,11),(10,12),(13,15),(14,16)$ & 2 & $(1,6)$ \\
\hline 3 & $(1,6),(1,16),(2,15),(4,7),(6,11),(5,12),(11,16),(10,13)$ & $2 \gamma^{2}+2+4 \gamma \cos \theta$ & $(1,8)$ \\
\hline 4 & $(1,8),(2,7),(2,13),(3,16),(6,9),(7,12),(11,14),(12,13)$ & $2 \gamma^{2}+2+4 \gamma \sin \theta$ & $(3,6)$ \\
\hline 5 & $(1,14),(4,15),(3,6),(4,5),(8,11),(6,10),(9,16),(10,15)$ & $2 \gamma^{2}+2-4 \gamma \sin \theta$ & $(2,5)$ \\
\hline 6 & $(2,5),(3,8),(3,14),(4,13),(7,10),(8,9),(12,15),(9,14)$ & $2 \gamma^{2}+2-4 \gamma \cos \theta$ & $(1,5)$ \\
\hline 7 & $(1,5),(1,13),(4,8),(2,6),(2,14),(3,7),(3,15),(4,16)$, & & $(1,9)$ \\
\hline & $(6,10),(7,11),(8,12),(5,9),(12,16),(11,15),(10,14),(9,13)$ & & $(1,7)$ \\
\hline 8 & $(1,9),(2,10),(3,11),(4,12),(6,14),(5,13),(7,15),(8,16)$ & $4 \gamma^{2}$ & $(4,6)$ \\
\hline 9 & $(1,7),(2,16),(6,12),(11,13)$ & $2 \gamma^{2}+4+4 \gamma \cos \theta+4 \gamma \sin \theta$ & $(2,8)$ \\
\hline 10 & $(1,15),(4,6),(5,11),(10,16)$ & $2 \gamma^{2}+4+4 \gamma \cos \theta-4 \gamma \sin \theta$ & $(3,5)$ \\
\hline 11 & $(3,13),(2,8),(7,9),(12,14))$ & $2 \gamma^{2}+4-4 \gamma \cos \theta+4 \gamma \sin \theta$ & $(1,12)$ \\
\hline 12 & $(3,5),(8,10),(9,15),(4,14)$ & $2 \gamma^{2}+4-4 \gamma \cos \theta-4 \gamma \sin \theta$ & $(1,10)$ \\
\hline 13 & $(1,12),(2,11),(6,13),(7,16)$ & $4 \gamma^{2}+2+4 \gamma \cos \theta+4 \gamma \sin \theta$ & $(1,1)$ \\
\hline 14 & $(1,10),(6,15),(4,11),(5,16)$ & $4 \gamma^{2}+2+4 \gamma \cos \theta-4 \gamma \sin \theta$ & $(1,10)$ \\
\hline 15 & $(2,9),(3,12),(7,14),(8,13)$ & $4 \gamma^{2}+2-4 \gamma \cos \theta+4 \gamma \sin \theta$ & $(2,9)$ \\
\hline 16 & $(3,10),(8,15),(4,9),(5,14)$ & $4 \gamma^{2}+2-4 \gamma \cos \theta-4 \gamma \sin \theta$ & $(3,10)$ \\
\hline 17 & $(1,11),(6,16)$ & $4 \gamma^{2}+4+8 \gamma \cos \theta$ & $(1,11)$ \\
\hline 18 & $(2,12),(7,13)$ & $4 \gamma^{2}+4+8 \gamma \sin \theta$ & $(2,12)$ \\
\hline 19 & $(3,9),(8,14)$ & $4 \gamma^{2}+4-8 \gamma \cos \theta$ & $(3,9)$ \\
\hline 20 & $(4,10),(5,15)$ & $4 \gamma^{2}+4-8 \gamma \sin \theta$ & $(4,10)$ \\
\hline
\end{tabular}

Now, from $(9), d_{\mathcal{C}_{l^{\prime}}}(\gamma, \theta)$ is minimum among all $d_{\mathcal{C}_{i}}(\gamma, \theta)$ for Step 2 For the singular fade state $\left(\gamma_{1}, \theta_{1}\right)$ in $\mathcal{H}_{W}$, identify the all values of $(\gamma, \theta) \neq\left(\gamma^{\prime}, \theta^{\prime}\right)$.

Definition 5: The region corresponding to distance class $\mathcal{C}$, $\mathcal{R}(\mathcal{C})$ denotes the region in the complex plane $\{(\Gamma, \Theta) / \mathcal{H}\}$ for which the class distance function $d_{\mathcal{C}}(\gamma, \theta)$ gives the minimum distance in $\mathcal{S}_{\text {eff }}$, i.e.,

$$
\begin{aligned}
& \mathcal{R}(\mathcal{C})=\{(\gamma, \theta) \in\{(\Gamma, \Theta) / \mathcal{H}\} \mid \\
&\left.d_{\mathcal{C}}(\gamma, \theta) \leq d_{\mathcal{C}^{\prime}}(\gamma, \theta) \text { for all } \mathcal{C}^{\prime} \neq \mathcal{C} \in \overline{\mathcal{C}}\right\} .
\end{aligned}
$$

Definition 6: When both the users use $M$-PSK constellations at the input, $\mathcal{R}_{W}(\mathcal{C})$ denotes the portion of the region $\mathcal{R}(\mathcal{C})$ lying in the wedge $[0, \pi / M]$, i.e.,

$$
\mathcal{R}_{W}(\mathcal{C})=\mathcal{R}(\mathcal{C}) \cap \text { wedge }[0, \pi / M] .
$$

Note that, when both the users use $M$-PSK constellations at the input, for some $\mathcal{C} \in \overline{\mathcal{C}}$ the corresponding region $\mathcal{R}(\mathcal{C})$ can be a null set, because the associated class distance function $d_{\mathcal{C}}(\gamma, \theta)$ does not give the minimum distance in $\mathcal{S}_{\text {eff }}$ for any value of $(\gamma, \theta)$ in $\{(\Gamma, \Theta) \backslash \mathcal{H}\}$. There is always a distance class $\mathcal{C} \in \overline{\mathcal{C}}$ for which the associated class distance function is $d_{\mathcal{C}(\gamma, \theta)}=d_{\min }(\mathcal{S})$. We denote this particular distance class as $\mathcal{C}_{d_{\min }(\mathcal{S})}$. From Lemma 1 the value of this class distance function is the upper bound for the minimum distance in $\mathcal{S}_{\text {eff }}$. For example when both users use QPSK signal sets at the input, then from Table I $\mathcal{C}_{d_{\min }(\mathcal{S})}=\mathcal{C}_{1}$ and the associated class distance function is $d_{\mathcal{C}_{1}}(\gamma, \theta)=d_{\min }(\mathcal{S})=\sqrt{2}$.

The procedure to obtain the quantization of the $(\Gamma, \Theta)$ plane, when both users use $M$-PSK constellations at the input, is as follows:

Step 1 Obtain the $N_{W}$ singular fade states in $\mathcal{H}_{W}$ i.e., lying in the wedge $[0, \pi / M]$. Each of these singular fade state is denoted by $\left(\gamma_{i}, \theta_{i}\right)$ where $1 \leq i \leq N_{W}$. set of class distance functions in $d_{\overline{\mathcal{C}}}(\gamma, \theta)$ that reduces to zero at that singular fade state $\left(\gamma_{1}, \theta_{1}\right)$. Choose the one among them, which is minimum in that set for all values of $(\gamma, \theta) \neq\left(\gamma_{1}, \theta_{1}\right)$. (From Lemma 4 there is always only one such class distance function.) Let this class distance function be $d_{\mathcal{C}_{k_{1}}}(\gamma, \theta)$ corresponding to distance class $\mathcal{C}_{k_{1}}$. Repeat this for all $\left(\gamma_{i}, \theta_{i}\right) \in \mathcal{H}_{W}$, to obtain a set of class distance functions $\left\{d_{\mathcal{C}_{k_{i}}}(\gamma, \theta)\right.$, $\left.1 \leq i \leq N_{W}\right\}$. Each $d_{\mathcal{C}_{k_{i}}}(\gamma, \theta)$ reduces to zero at the singular fade state $\left(\gamma_{i}, \theta_{i}\right)$. This is the set of all possible class distance functions other than $d_{\min }(\mathcal{S})$, that can possibly produce the minimum distance in $\mathcal{S}_{\text {eff }}$.

Step 3 To find the region $\mathcal{R}_{W}\left(\mathcal{C}_{k_{i}}\right)$, we need to obtain the values of $(\gamma, \theta) \in$ wedge $[0, \pi / M]$ for which $d_{\mathcal{C}_{k_{i}}}^{2}(\gamma, \theta) \leq$ $d_{\mathcal{C}_{k_{j}}}^{2}(\gamma, \theta)$ where $1 \leq j \neq i \leq N_{W}$, and $d_{\mathcal{C}_{k_{i}}}^{2}(\gamma, \theta) \leq$ $d_{\text {min }}^{2}(\mathcal{S})$. The curves $d_{\mathcal{C}_{k_{i}}}^{2}(\gamma, \theta)=d_{\mathcal{C}_{k_{j}}}^{2}(\gamma, \theta), 1 \leq i \neq$ $j \leq N_{W}$, form the pairwise boundary between the regions corresponding to the two distance classes $\mathcal{C}_{k_{i}}$ and $\mathcal{C}_{k_{j}}$. The curves $d_{\mathcal{C}_{k_{i}}}^{2}(\gamma, \theta)=d_{\text {min }}^{2}(\mathcal{S})$ form the pairwise boundary between the regions corresponding to

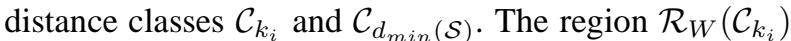
is that region in the wedge $[0, \pi / M]$ excluding the complex point $\left(\gamma_{i}, \theta_{i}\right)$, which is the innermost region bounded by these pairwise boundaries, enclosing the singular fade state $\left(\gamma_{i}, \theta_{i}\right)$. For example, Fig. 6 depicts the region corresponding to the singular fade state at $(1,0)$ when both users use 8-PSK signal sets. In the figure, the curve $d_{1}^{2}=d_{j}^{2}$ refers to the curve $d_{\mathcal{C}_{k_{1}}}^{2}(\gamma, \theta)=$ $d_{\mathcal{C}_{k_{k}}}^{2}(\gamma, \theta)$, and $d_{1}^{2}=2-\sqrt{2}$ refers to the curve $d_{\mathcal{C}_{k_{1}}}^{2}(\gamma, \theta)=2-\sqrt{2}$. It is the innermost region (shaded in the figure) in the wedge $[0, \pi / 8]$ bounded by the pair- 


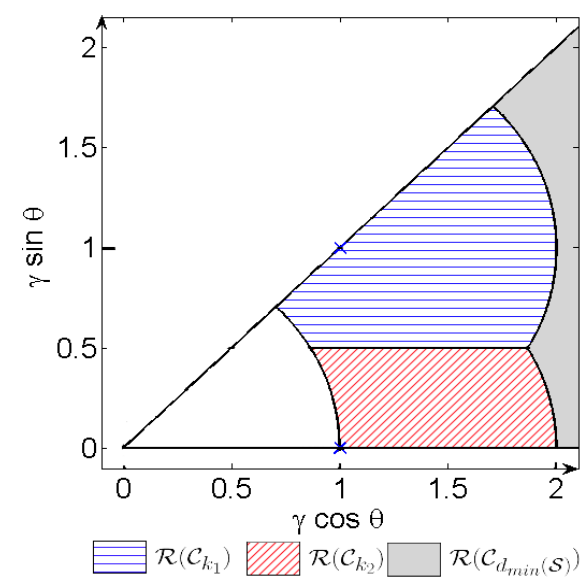

Fig. 3: Quantization of the wedge $[0, \pi / 4]$ for QPSK signal sets.

wise boundaries $d_{\mathcal{C}_{k_{1}}}^{2}(\gamma, \theta)=d_{\mathcal{C}_{k_{2}}}^{2}(\gamma, \theta), d_{\mathcal{C}_{k_{1}}}^{2}(\gamma, \theta)=$ $d_{\mathcal{C}_{k_{4}}}^{2}(\gamma, \theta), d_{\mathcal{C}_{k_{1}}}^{2}(\gamma, \theta)=d_{\mathcal{C}_{k_{5}}}^{2}(\gamma, \theta)$ and $d_{\mathcal{C}_{k_{1}}}^{2}(\gamma, \theta)=$ $d_{\mathcal{C}_{k_{6}}}^{2}(\gamma, \theta)$ surrounding the singular fade state $(1,0)$. Once the regions $\mathcal{R}\left(\mathcal{C}_{k_{i}}\right), 1 \leq i \leq N_{W}$ are obtained, the region exterior to all these regions, lying within the wedge $[0, \pi / M]$, is the region where $d_{\min }(\mathcal{S})$ is the minimum distance in $\mathcal{S}_{\text {eff }}$, i.e., the region $\mathcal{R}_{W}\left(\mathcal{C}_{d_{\text {min }}(\mathcal{S})}\right)$.

Step 4 The quantization obtained in Step 3, for the wedge $[0, \pi / M]$, can now be extended by the procedure suggested in Section $\amalg-A$ to cover the entire $(\Gamma, \Theta)$ plane.

We will illustrate the procedure with two examples.

Example 3: Channel Quantization for QPSK signal sets. Here we consider the scenario where both users use QPSK constellations at input, i.e. $M=4$. From Lemma 3, there are two singular fade states in the wedge $[0, \pi / 4]$, i.e. $N_{W}=2$ and these are at $(1,0)$ and $\left(\sqrt{2}, \frac{\pi}{4}\right)$. The class distance functions in $d_{\overline{\mathcal{C}}}(\gamma, \theta)$ which reduce to zero at these singular fade states are identified. For the singular fade state $\left(\sqrt{2}, \frac{\pi}{4}\right)$ the distance $d_{\mathcal{C}_{k_{1}}}$ falls to zero, and for $(1,0)$ the distances $d_{\mathcal{C}_{k_{2}}}$ and $d_{\mathcal{C}_{k_{3}}}$ both fall to zero, as shown in Fig 2 These are as follows:

$$
\begin{aligned}
& d_{\mathcal{C}_{k_{1}}}^{2}(\gamma, \theta)=2 \gamma^{2}+4-4 \gamma \cos \theta-4 \gamma \sin \theta \\
& d_{\mathcal{C}_{k_{2}}}^{2}(\gamma, \theta)=2 \gamma^{2}+2-4 \gamma \cos \theta \\
& d_{\mathcal{C}_{k_{3}}}^{2}(\gamma, \theta)=4 \gamma^{2}+4-8 \gamma \cos \theta=2 d_{\mathcal{C}_{k_{2}}}^{2} \geq d_{\mathcal{C}_{k_{2}}}^{2} .
\end{aligned}
$$

As $d_{\mathcal{C}_{k_{3}}}(\gamma, \theta)>d_{\mathcal{C}_{k_{2}}}(\gamma, \theta)$ for all values of $(\gamma, \theta) \neq(1,0)$, we consider the class distance function corresponding to the singular fade state $(1,0)$ as $d_{\mathcal{C}_{k_{2}}}(\gamma, \theta)$. Now we proceed to obtain the regions $\mathcal{R}_{W}\left(\mathcal{C}_{k_{1}}\right), \mathcal{R}_{W}\left(\mathcal{C}_{k_{2}}\right)$ and $\mathcal{R}_{W}\left(\mathcal{C}_{d_{\text {min }}(\mathcal{S})}\right)$. The pair-wise boundaries are obtained as follows,

$$
\begin{aligned}
& d_{\mathcal{C}_{k_{1}}}^{2}(\gamma, \theta)=d_{\mathcal{C}_{\mathcal{L}_{2}}}^{2}(\gamma, \theta) \Rightarrow \gamma \sin \theta=1 / 2 \\
& d_{\mathcal{C}_{k_{1}}}^{2}(\gamma, \theta)=d_{\min }^{2}(\mathcal{S})=2 \Rightarrow(\gamma \cos \theta-1)^{2}+(\gamma \sin \theta-1)^{2}=1 \\
& d_{\mathcal{C}_{k_{2}}}^{2}(\gamma, \theta)=d_{\text {min }}^{2}(\mathcal{S})=2 \Rightarrow(\gamma \cos \theta-1)^{2}+\gamma^{2} \sin ^{2} \theta=1 .
\end{aligned}
$$

These regions are shown in Fig. 3 for the wedge $[0, \pi / 4]$. This can now be extended to cover the entire range of $\theta$. For this,

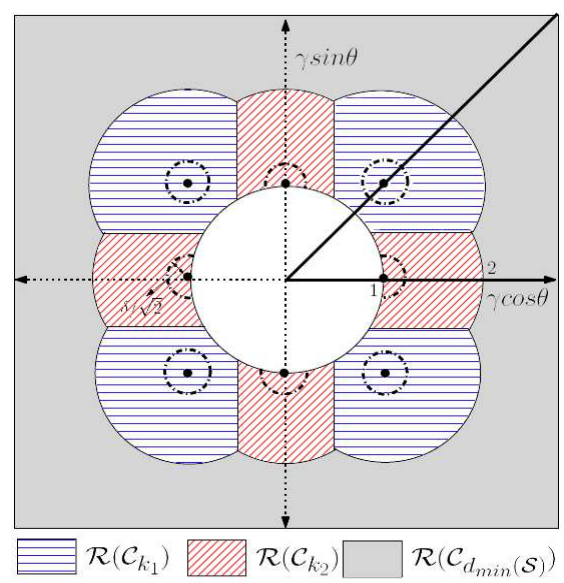

Fig. 4: Quantization of the entire $(\Gamma, \Theta)$ plane for QPSK signal sets

the quantization obtained for $\theta \in[0, \pi / 4]$ is reflected along the line $\theta=\pi / 4$ to obtain the quantization for the wedge $[0, \pi / 2]$. This is now rotated by $\frac{k \pi}{2}, 1 \leq k \leq 3$, to obtain the quantization for $\theta \in\left[\frac{k \pi}{2}, \frac{(k+1) \pi}{2}\right]$, thus covering the entire $(\Gamma, \Theta)$ plane. This has been shown in Fig. 4.

Example 4: Channel quantization for 8-PSK signal sets Here we consider the scenario when both users use 8-PSK signal sets at the input. The number of singular fade states lying in the wedge $[0, \pi / 8]$ is, $N_{W}=7$. The singular fade states $\left(\gamma_{i}, \theta_{i}\right), 1 \leq i \leq 7$ are as follows:

$$
\begin{aligned}
& (1,0),(\sqrt{2}, 0),(1+\sqrt{2}, 0), \\
& \left(\sqrt{4-2 \sqrt{2}}, \frac{\pi}{8}\right),\left(\sqrt{1+\frac{1}{\sqrt{2}}}, \frac{\pi}{8}\right), \\
& \left(\sqrt{2+\sqrt{2}}, \frac{\pi}{8}\right),\left(\sqrt{4+2 \sqrt{2}}, \frac{\pi}{8}\right) .
\end{aligned}
$$

The class distance functions in $d_{\overline{\mathcal{C}}}(\gamma, \theta)$ which reduce to zero for each of the above singular fade states are identified. When more than one class distance function reduces to zero at a singular fade state $\left(\gamma_{i}, \theta_{i}\right)$, the one which is minimum among them for any other $(\gamma, \theta) \neq\left(\gamma_{i}, \theta_{i}\right)$ is chosen. The class distance functions $d_{\mathcal{C}_{k_{i}}}(\gamma, \theta), 1 \leq i \leq 7$ are identified as shown in Fig. 5 In the figure $d_{i}$ denotes the class distance function $d_{\mathcal{C}_{k_{i}}}(\gamma, \theta)$. These are as follows

$$
\begin{aligned}
& d_{\mathcal{C}_{k_{1}}}^{2}(\gamma, \theta)=(2-\sqrt{2})\left(\gamma^{2}-2 \gamma \cos \theta+1\right) \\
& d_{\mathcal{C}_{k_{2}}}^{2}(\gamma, \theta)=2 \gamma^{2}+4-4 \sqrt{2} \gamma \cos \theta \\
& d_{\mathcal{C}_{k_{3}}}^{2}(\gamma, \theta)=(2-\sqrt{2}) \gamma^{2}+2+\sqrt{2}-2 \sqrt{2} \gamma \cos \theta \\
& d_{\mathcal{C}_{k_{4}}}^{2}(\gamma, \theta)=(2+\sqrt{2}) \gamma^{2}+4-2(2+\sqrt{2}) \gamma \cos \theta-2 \sqrt{2} \gamma \sin \theta \\
& d_{\mathcal{C}_{k_{5}}}^{2}(\gamma, \theta)=2 \gamma^{2}+2+\sqrt{2}-2(1+\sqrt{2}) \gamma \cos \theta-2 \gamma \sin \theta \\
& d_{\mathcal{C}_{k_{6}}}^{2}(\gamma, \theta)=(2-\sqrt{2}) \gamma^{2}+2-2(\sqrt{2}-1) \gamma \sin \theta-2 \gamma \cos \theta \\
& d_{\mathcal{C}_{k_{7}}}^{2}(\gamma, \theta)=(2-\sqrt{2}) \gamma^{2}+4-2(2-\sqrt{2}) \gamma \sin \theta-2 \sqrt{2} \gamma \cos \theta .
\end{aligned}
$$

Now to obtain the region $\mathcal{R}_{W}\left(\mathcal{C}_{k_{1}}\right)$ we need to obtain the 


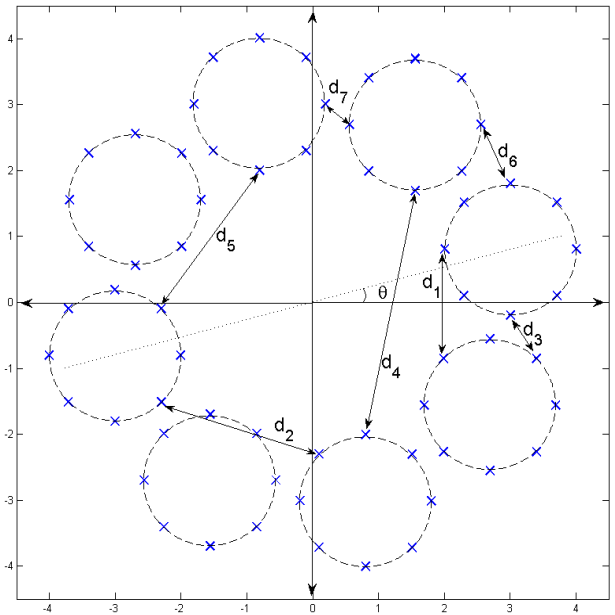

Fig. 5: Effective constellation $\mathcal{S}_{\text {eff }}$ for $(\gamma, \theta)=\left(2.9,10^{\circ}\right)$ when both users use 8-PSK signal set.

curves $d_{\mathcal{C}_{k_{1}}}^{2}(\gamma, \theta)=d_{\mathcal{C}_{k_{j}}}^{2}(\gamma, \theta), 2 \leq j \leq 7$ and $d_{\mathcal{C}_{k_{1}}}^{2}(\gamma, \theta)=$ $d_{\min }^{2}(\mathcal{S})=2-\sqrt{2}$. These are as follows:

$$
\begin{aligned}
& d_{\mathcal{C}_{k_{1}}}^{2}(\gamma, \theta)=d_{\mathcal{C}_{k_{2}}}^{2}(\gamma, \theta) \Rightarrow(\gamma \cos \theta-(3-\sqrt{2}))^{2}+\gamma^{2} \sin ^{2} \theta=10-7 \sqrt{2} \\
& d_{\mathcal{C}_{\mathcal{K}_{1}}}^{2}(\gamma, \theta)=d_{\mathcal{C}_{k_{3}}}^{2}(\gamma, \theta) \Rightarrow \gamma \cos \theta=\frac{1}{2-\sqrt{2}} \\
& d_{\mathcal{C}_{k_{1}}}^{2}(\gamma, \theta)=d_{\mathcal{C}_{k_{4}}}^{2}(\gamma, \theta) \Rightarrow(\gamma \cos \theta-1)^{2}+\left(\gamma \sin \theta-\frac{1}{2}\right)^{2}=\frac{3-2 \sqrt{2}}{4} \\
& d_{\mathcal{C}_{k_{1}}}^{2}(\gamma, \theta)=d_{\mathcal{C}_{k_{5}}}^{2}(\gamma, \theta) \Rightarrow\left(\gamma \cos \theta-2+\frac{1}{\sqrt{2}}\right)^{2}+\left(\gamma \sin \theta-\frac{1}{\sqrt{2}}\right)^{2} \\
& d_{\mathcal{C}_{k_{1}}}^{2}(\gamma, \theta)=3-2 \sqrt{2} \\
& d_{\mathcal{C}_{\mathcal{C}_{1}}}^{2}(\gamma, \theta)=d_{\mathcal{C}_{k_{6}}}^{2}(\gamma, \theta) \Rightarrow \gamma \cos \theta+\gamma \sin \theta=1+\frac{1}{\sqrt{2}} \\
& d_{\mathcal{C}_{\mathcal{C}_{1}}}^{2}(\gamma, \theta)=2-\sqrt{2} \Rightarrow(\gamma \cos \theta-1)^{2}+\gamma^{2} \sin ^{2} \theta=1 .
\end{aligned}
$$

All of the above curves are shown in Fig. 6. In the figure, the curve $d_{1}^{2}=d_{j}^{2}$ refers to the curve $d_{\mathcal{C}_{k_{1}}}^{2}(\gamma, \theta)=d_{\mathcal{C}_{k_{k}}}^{2}(\gamma, \theta)$, and $d_{1}^{2}=2-\sqrt{2}$ refers to the curve $d_{\mathcal{C}_{k_{1}}}^{2}(\gamma, \theta)=2-\sqrt{2}$. The region $\mathcal{R}_{W}\left(\mathcal{C}_{k_{1}}\right)$ is the innermost region in the wedge $[0, \pi / 8]$ bounded by these curves, surrounding the point $(1,0)$. It is the shaded region in the Fig. 6 All the regions $\mathcal{R}_{W}\left(\mathcal{C}_{k_{i}}\right), 1 \leq i \leq$ 7 can be obtained by the same procedure. The region exterior to all these regions, inside the wedge $[0, \pi / 8]$ is the region $\mathcal{R}_{W}\left(\mathcal{C}_{d_{\text {min }}(\mathcal{S})}\right)$, where $d_{\text {min }}(\mathcal{S})=\sqrt{2-\sqrt{2}}$ is the minimum distance in $\mathcal{S}_{\text {eff }}$. Thus, the quantization of the fade states for the wedge $[0, \pi / 8]$ is obtained. It is shown is Fig. 7 in the next page. This can now be extended to cover the entire $(\Gamma, \Theta)$ plane by the similar technique used for the QPSK case in the Example 3

\section{THE ADAPTIVE MODULATION SCHEME}

In this section the fade states which results in reducing the minimum distance in $\mathcal{S}_{\text {eff }}$ below a minimum distance guarantee of $\delta$ are identified. Then a modulation scheme is proposed for the users to avoid these fade states by suitable relative rotation between the signal sets used by the two users.

\section{A. Adaptive Modulation Scheme}

It is clear from Section II-B that the minimum distance in $\mathcal{S}_{\text {eff }}$ falls to zero at the singular fade states. For fade states $(\gamma, \theta)$ lying close to a singular fade state, the minimum distance in $\mathcal{S}_{\text {eff }}$ is very low, resulting in degradation of error performance at the destination. Hence, such values of $(\gamma, \theta)$ have to be avoided to provide better performance.

Our goal is to provide a minimum distance guarantee of $\delta$ in $\mathcal{S}_{\text {eff }}$ i.e., not allow minimum distance in $\mathcal{S}_{\text {eff }}$ to fall below $\delta$. In the previous subsection, the regions $\mathcal{R}\left(\mathcal{C}_{k_{i}}\right)$ on the $(\Gamma, \Theta)$ plane was identified, in which the class distance function $d_{\mathcal{C}_{k_{i}}}(\gamma, \theta), 1 \leq i \leq N_{W}$ gives the minimum distance in $\mathcal{S}_{\text {eff }}$. In order to satisfy the minimum distance guarantee of $\delta$ in $\mathcal{S}_{\text {eff }}$, it is thus required to avoid the fade states $(\gamma, \theta)$ for which $d_{\mathcal{C}_{k_{i}}}(\gamma, \theta)<\delta, 1 \leq i \leq N_{W}$.

At the singular fade state $\left(\gamma_{i}, \theta_{i}\right)$, the class distance function $d_{\mathcal{C}_{k_{i}}}(\gamma, \theta)$ reduces to zero. If $\left\{\left(s_{1, i}, s_{2, i}\right),\left(s_{1, i}^{\prime}, s_{2, i}^{\prime}\right)\right\}$ is the representative element for the distance class $\mathcal{C}_{k_{i}}$, then at the singular fade state $\left(\gamma_{i}, \theta_{i}\right)$ the two points $\left(s_{1, i}, s_{2, i}\right)$ and $\left(s_{1, i}^{\prime}, s_{2, i}^{\prime}\right)$ collapse to a single point in $\mathcal{S}_{\text {eff }}$. From the definition of singular fade state (4), we have

$$
\gamma_{i} e^{j \theta_{i}}=-\frac{s_{1, i}-s_{1, i}^{\prime}}{s_{2, i}-s_{2, i}^{\prime}}
$$

From (3), we have

$$
d_{\mathcal{C}_{k_{i}}}(\gamma, \theta)=\left|s_{2, i}-s_{2, i}^{\prime}\right|\left|\gamma e^{j \theta}-\gamma_{i} e^{j \theta_{i}}\right| .
$$

Only those fade states $(\gamma, \theta)$ which results in $d_{\mathcal{C}_{k_{i}}}(\gamma, \theta)<\delta$, $1 \leq i \leq N_{W}$ have to be avoided, i.e., from (10), we need to avoid the fade states $(\gamma, \theta)$, for $1 \leq i \leq N_{W}$, where

$$
\left|\gamma e^{j \theta}-\gamma_{i} e^{j \theta_{i}}\right|<\frac{\delta}{\left|s_{2, i}-s_{2, i}^{\prime}\right|} .
$$

The above equation represents a circular region in the complex plane $(\Gamma, \Theta)$ centred at the singular fade state $\left(\gamma_{i}, \theta_{i}\right)$ and radius $\delta /\left|s_{2, i}-s_{2, i}^{\prime}\right|$. We call these circular regions, the violation circles because when the fade state lies inside them the minimum distance requirement of $\mathcal{S}_{\text {eff }}$ is violated. For fade states outside the violation circles the minimum distance in $\mathcal{S}_{\text {eff }}$ is always greater than $\delta$. The radius of the violation circle centred at the singular fade state $\left(\gamma_{i}, \theta_{i}\right)$ by is denoted by $\rho\left(\gamma_{i}, \theta_{i}\right)$. Formally violation circles are defined as follows:

Definition 7: Violation Circles are circular regions on the $(\Gamma, \Theta)$ plane with centres at the singular fade states $\left(\gamma_{i}, \theta_{i}\right)=$ $-\frac{s_{1, i}-s_{1, i}^{\prime}}{s_{2, i}-s_{2, i}^{\prime}}$ with radius $\rho\left(\gamma_{i}, \theta_{i}\right)=\delta /\left|s_{2, i}-s_{2, i}^{\prime}\right|$ for $1 \leq i \leq$ $N_{W}$.

It is observed that the the violation circles centred at $\left(\gamma_{i}, \theta_{i}+p \frac{2 \pi}{M}\right), 1 \leq i \leq N_{W}$ and $1 \leq p \leq M-1$ have the same radius as the one centred at $\left(\gamma_{i}, \theta_{i}\right)$, where $\left(\gamma_{i}, \theta_{i}\right) \in \mathcal{H}_{W}$. This is because from Lemma 2, the corresponding effective constellations are the same.

Example 5: When both users use QPSK constellations at the input, the violation circles are as follows. At singular fade state $(\sqrt{2}, \pi / 4)$ the class distance function $d_{\mathcal{C}_{k_{1}}}(\gamma, \theta)$ reduces to zero, i.e. the points 3 and 5 in $\mathcal{S}_{\text {eff }}$ collapse to a single point, 


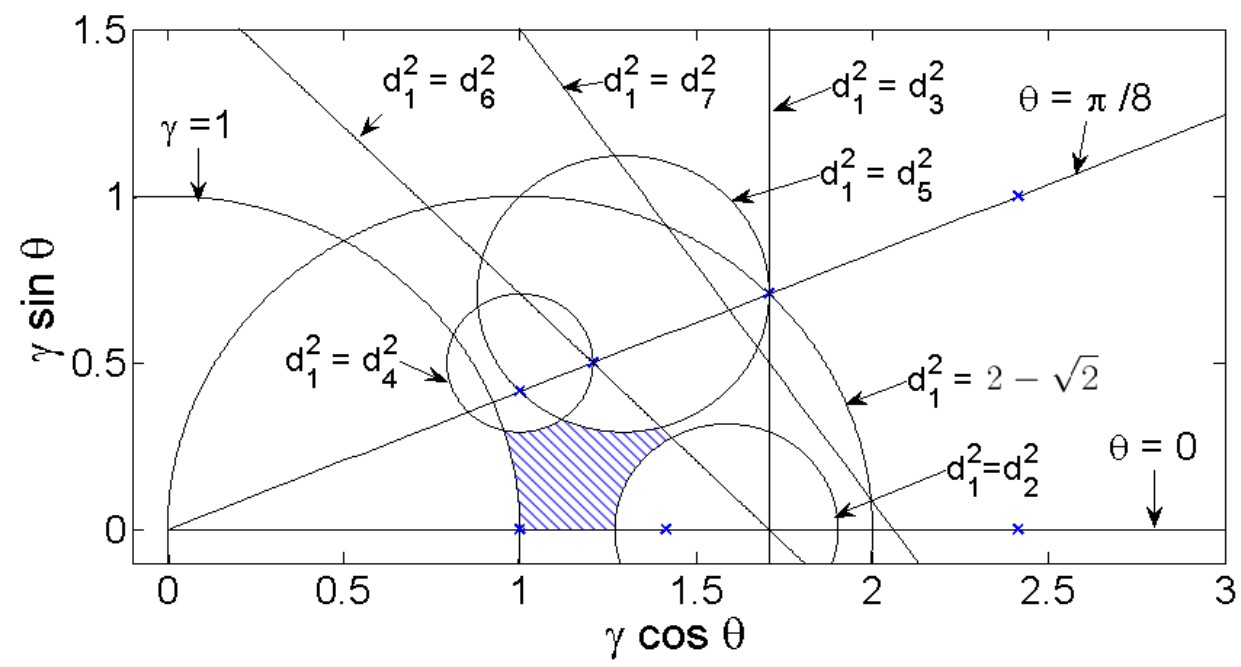

Fig. 6: The figure explains how to region $\mathcal{R}\left(d_{1}\right)$ (shaded region in the figure) corresponding to the singular fade state $\left(1,0^{\circ}\right)$ is obtained.

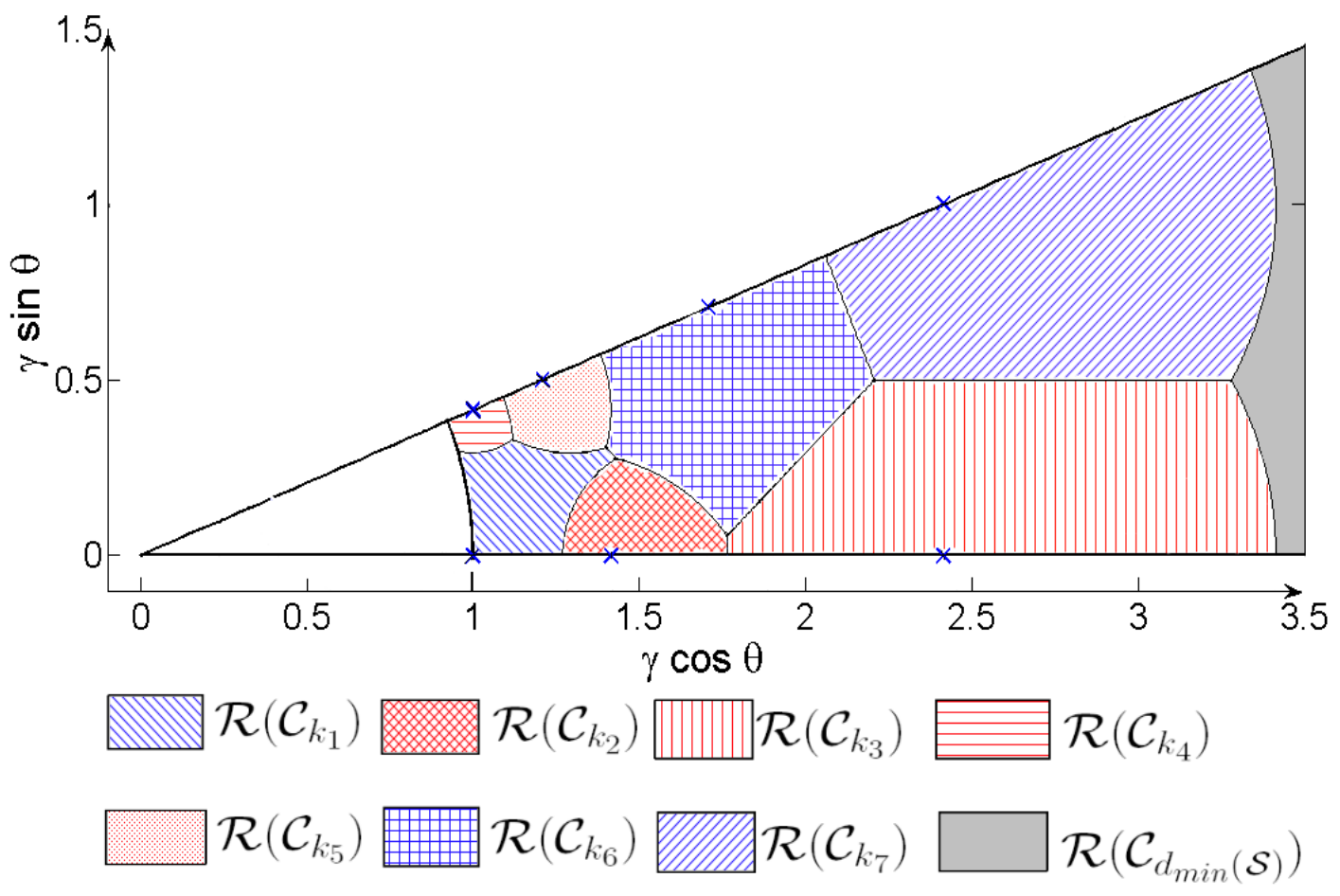

Fig. 7: Channel quantization for $\theta \in[0, \pi / 8]$ when both users use 8 -PSK signal sets

as shown in Fig. 2. The 3 and 5 are obtained after combining $3,1 \in \mathcal{S}$ and $1,2 \in \mathcal{S}$ respectively. Thus, $\left|s_{2,1}-s_{2,1}^{\prime}\right|=\sqrt{2}$. The violation circle corresponding to class distance function $d_{\mathcal{C}_{k_{1}}}(\gamma, \theta)$ is the circular region centred at $(\sqrt{2}, \pi / 4)$ and radius $\delta / \sqrt{2}$. Similarly, for the singular fade state at $(1,0)$ the violation circle has a radius of $\delta / \sqrt{2}$. These are shown by dotted circles around the singular fade states in Fig. 4

Example 6: When both users are using 8-PSK constellations at the input, the centres and radii of the violation circles corresponding to the distances $d_{\mathcal{C}_{k_{i}}}, 1 \leq i \leq 7$ are tabulated in Table [I]

When the fade state $(\gamma, \theta)$ lies inside any of the violation circles the users need to adapt their transmission, in order to avoid these fade states effectively. One way to achieve this without increasing the transmit power, is to rotate the constellation of User-2. Rotation can be interpreted as simply altering the phase of the fade state.

Lemma 5: When the fade state is $(\gamma, \theta)$, rotation of the constellation of User-2 by an angle $\alpha$ with respect to the constellation of User-1 in an anticlockwise direction, results 
TABLE II: Centre and Radius of violation circles for 8-PSK case

\begin{tabular}{|c|l|c|}
\hline$i$ & Centre & Radius \\
\hline 1 & $(1,0)$ & $\frac{\delta}{\sqrt{2-\sqrt{2}}}$ \\
\hline 2 & $(\sqrt{2}, 0)$ & $\frac{\delta}{\sqrt{2}}$ \\
\hline 3 & $(\sqrt{2}+1,0)$ & $\frac{\delta}{\sqrt{2-\sqrt{2}}}$ \\
\hline 4 & $\left(\sqrt{4-2 \sqrt{2}}, \frac{\pi}{8}\right)$ & $\frac{\delta}{\sqrt{2+\sqrt{2}}}$ \\
\hline 5 & $\left(\sqrt{1+\frac{1}{\sqrt{2}}}, \frac{\pi}{8}\right)$ & $\frac{\delta}{\sqrt{2}}$ \\
\hline 6 & $\left(\sqrt{2+\sqrt{2}}, \frac{\pi}{8}\right)$ & $\frac{\delta}{\sqrt{2-\sqrt{2}}}$ \\
\hline 7 & $\left(\sqrt{4+2 \sqrt{2}}, \frac{\pi}{8}\right)$ & $\frac{\delta}{\sqrt{2-\sqrt{2}}}$ \\
\hline
\end{tabular}

in effectively altering the phase of the fade state from $\theta$ to $\theta+\alpha$.

Proof: Let $\mathcal{S}$ be the constellation being used by both the users at the input. Now the User-2 rotates its constellation by angle $\alpha$ in the anticlockwise direction, such that it can now be represented as $e^{j \alpha} \mathcal{S}$. The effective constellation $\mathcal{S}_{\text {eff }}$ can be written as

$$
\mathcal{S}+\gamma e^{j \theta}\left\{e^{j \alpha} \mathcal{S}\right\}=\mathcal{S}+\gamma e^{j(\theta+\alpha)} \mathcal{S} .
$$

Hence, the fade state $(\gamma, \theta)$ is transformed to $(\gamma, \theta+\alpha)$ after rotation.

The proposed strategy is thus, to rotate the signal set of User-2 whenever the fade state $(\gamma, \theta)$ lies within any of the violation circles such that the transformed fade state lies outside the violation circles, in order to satisfy the minimum distance guarantee in $\mathcal{S}_{\text {eff }}$. For fade states outside the violation circles no rotation is required. The destination which has complete CSI sends feedback of $\left\lceil\log _{2}\left(N_{W}+1\right)\right\rceil$ bits to the users to indicate in which one of the violation circles the fade state lies, or if it lies outside all of them.

\section{B. Optimal Angle of Rotation for the M-PSK case}

Definition 8: An optimal rotation angle, for a violation circle with centre at singular fade state $\left(\gamma_{i}, \theta_{i}\right), 1 \leq i \leq N_{W}$ is that angle of rotation which maximizes the minimum distance in $\mathcal{S}_{\text {eff }}$ for the same transmit power, when fade state $(\gamma, \theta)=\left(\gamma_{i}, \theta_{i}\right)$.

It should be noted for non-singular fade states inside the violation circle, the minimum distance in $\mathcal{S}_{\text {eff }}$ after rotation will be less than what it could have been if the fade state $(\gamma, \theta)$ was exactly known at the users. When both users use $M$-PSK constellations at the input, it is sufficient to concentrate on the violation circles centred at $\left(\gamma_{i}, \theta_{i}\right) \in \mathcal{H}_{W}$, because the optimal rotation angles for these, is also optimal for other such circles centred at $\left(\gamma_{i}, \theta_{i}+p \frac{2 \pi}{M}\right)$, where $1 \leq p \leq M-1$. This follows from the fact that the corresponding effective constellations are equivalent.

From Lemma 5, rotation of the constellation of User-2 relative to User-1, results in effectively altering the phase of the fade state. Rotation thus results in moving the violation circle with centre at singular fade state $\left(\gamma_{i}, \theta_{i}\right)$, along a circular arc such that its centre always lie on the curve $\gamma=\gamma_{i}$ and within the wedge $[0, \pi / M]$. In order to obtain the optimal angle of rotation for the violation circle centred at $\left(\gamma_{i}, \theta_{i}\right)$, it is required to calculate for fixed $\gamma=\gamma_{i}$ the value of phase $\theta=\theta_{i, o p t}$, $\theta \in[0, \pi / M]$ which maximizes the minimum distance in $\mathcal{S}_{\text {eff. }}$ Thus after rotation the violation circle centred $\left(\gamma_{i}, \theta_{i}\right)$ is shifted such that its new centre is the point $\left(\gamma_{i}, \theta_{i, o p t}\right)$ on the $(\Gamma, \Theta)$ plane. We refer to this rotated violation circle as the effective shifted circle. We now prove two lemmas to obtain the value $\theta_{i, \text { opt }}$ when both users use $M$-PSK signal sets.

Lemma 6: Let $d_{\mathcal{C}_{k_{i}}}(\gamma, \theta)$ be the class distance function which reduces to zero at the singular fade state $\left(\gamma_{i}, \theta_{i}\right) \in \mathcal{H}_{W}$. For a fixed $\gamma_{0}$ and $\left(\gamma_{0}, \theta\right)$ lying within the wedge $[0, \pi / M]$, the value of $d_{\mathcal{C}_{k_{i}}}\left(\gamma_{0}, \theta\right)$ increases as the difference $\left|\theta_{i}-\theta\right|$ increases.

Proof: From (10), we have

$$
\begin{aligned}
d_{\mathcal{C}_{k_{i}}}^{2}\left(\gamma_{0}, \theta\right) & =\left|s_{2, i}-s_{2, i}^{\prime}\right|^{2}\left|\gamma_{0} e^{j \theta}-\gamma_{i} e^{j \theta_{i}}\right|^{2} \\
& =\left|s_{2, i}-s_{2, i}^{\prime}\right|^{2} \gamma_{0}^{2}\left|1-\gamma^{\prime} e^{j\left(\theta_{i}-\theta\right)}\right|^{2} \text { where } \gamma^{\prime}=\gamma_{i} / \gamma_{0}>0 \\
& =\left|s_{2, i}-s_{2, i}^{\prime}\right|^{2} \gamma_{0}^{2}\left\{1+\gamma^{\prime 2}-2 \gamma^{\prime} \cos \left|\theta_{i}-\theta\right|\right\} .
\end{aligned}
$$

As $\cos \phi$ is a decreasing function of $\phi, 0 \leq \phi \leq \pi / M$, $d_{\mathcal{C}_{k_{i}}}^{2}\left(\gamma_{0}, \theta\right)$ increases as $\left|\theta_{i}-\theta\right|$ increases. This proves the lemma.

Lemma 7: Let $\left(\gamma_{i}, \theta_{i}\right)$ be a singular fade state. Let $\mathcal{R}_{W}\left(\mathcal{C}_{k_{a}}\right)$ and $\mathcal{R}_{W}\left(\mathcal{C}_{k_{b}}\right)$ be the regions surrounding the singular fade states $\left(\gamma_{a}, \theta_{a}\right)$ and $\left(\gamma_{b}, \theta_{b}\right)$ respectively. Consider the arc traced by the point $\left(\gamma_{i}, \theta\right)$ that lies within the wedge $[0, \pi / M]$ as $\theta$ varies in the direction to move away from the singular fade state $\left(\gamma_{i}, \theta_{i}\right)$. Let the region $\mathcal{R}\left(\mathcal{C}_{k_{a}}\right)$ is encountered before $\mathcal{R}\left(\mathcal{C}_{k_{b}}\right)$ as $\theta$ varies. We have the following:

(i) The minimum distance in $\mathcal{S}_{\text {eff }}$ is maximized at one of the points of intersection of this arc and the boundaries between the regions $\mathcal{R}\left(\mathcal{C}_{k_{a}}\right)$ and $\mathcal{R}\left(\mathcal{C}_{k_{b}}\right)$.

(ii) Among all the points in (i), those that lie on the boundary between $\mathcal{R}\left(\mathcal{C}_{k_{a}}\right)$ and $\mathcal{R}\left(\mathcal{C}_{k_{b}}\right)$ with $\theta_{i}=\theta_{a}=\theta_{b}$ can never correspond to the maximum value.

Proof: The proof for part (i) is as follows. Let A and B be the first and the second points of intersection of the arc with the boundary of the region $\mathcal{R}_{W}\left(\mathcal{C}_{k_{a}}\right)$. From Section II-B, the value of class distance function $d_{\mathcal{C}_{k_{a}}}(\gamma, \theta)$ gives the minimum distance in $\mathcal{S}_{\text {eff }}$ when $(\gamma, \theta) \in \mathcal{R}_{W}\left(\mathcal{C}_{k_{a}}\right)$. Now if $\theta_{a}=\theta_{i}$, then from Lemma 6 $d_{\mathcal{C}_{k_{a}}}\left(\gamma_{i}, \theta\right)$ increases as $\left(\gamma_{i}, \theta\right)$ moves from $\mathbf{A}$ to $\mathbf{B}$. Likewise, if $\theta_{a} \neq \theta_{i}$, then again from Lemma 6. $d_{\mathcal{C}_{k_{a}}}\left(\gamma_{i}, \theta\right)$ decreases as one moves from $\mathbf{A}$ to $\mathbf{B}$. Thus the minimum distance in $\mathcal{S}_{\text {eff }}$ can never be maximum for $\left(\gamma_{i}, \theta\right)$ lying inside the regions $\mathcal{R}_{W}\left(\mathcal{C}_{k_{a}}\right)$. It can only be maximized at the points of intersection of the arc with the boundary of the region.

To prove the second part of the lemma, we assume $\theta_{i}=$ $\pi / M$. Consider the scenario shown in Fig. 8. To prove part (ii), we need to show that the minimum distance in $\mathcal{S}_{\text {eff }}$ can never be maximum at the point $\mathbf{B}$. In the region $\mathcal{R}_{W}\left(\mathcal{C}_{k_{a}}\right)$ the value of class distance function $d_{\mathcal{C}_{k_{a}}}(\gamma, \theta)$ gives the minimum distance in $\mathcal{S}_{\text {eff. }}$ As $\left(\gamma_{i}, \theta\right)$ moves from $\mathbf{A}$ to $\mathbf{B}$, from Lemma 6 , the minimum distance in $\mathcal{S}_{\text {eff }}$, i.e. the value of $d_{\mathcal{C}_{k_{a}}}\left(\gamma_{i}, \theta\right)$, increases since $\left|\theta_{a}-\theta\right|$ increases. At $\mathbf{B}$, we 


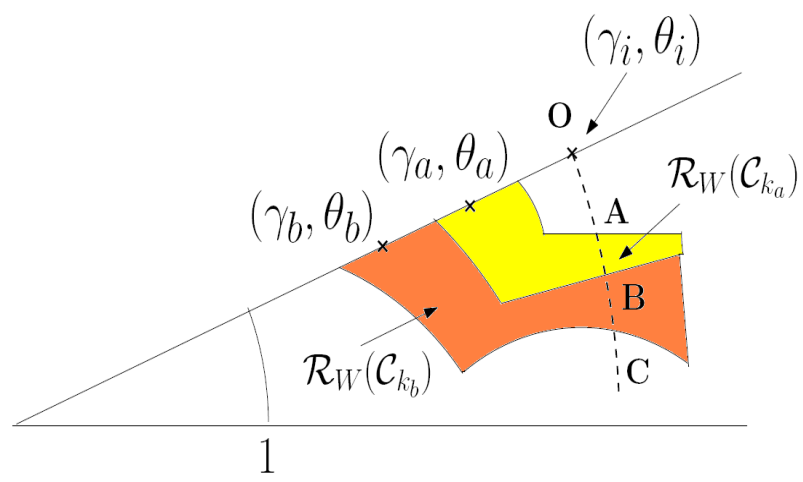

Fig. 8: Diagram illustrates the variation of the minimum distance in $\mathcal{S}_{\text {eff }}$, for fixed $\gamma_{i}$ on varying $\theta$

have $d_{\mathcal{C}_{k_{a}}}\left(\gamma_{i}, \theta\right)=d_{\mathcal{C}_{k_{b}}}\left(\gamma_{i}, \theta\right)$. Beyond $\mathbf{B}$, in the region $\mathcal{R}_{W}\left(\mathcal{C}_{k_{b}}\right)$, the class distance function $d_{\mathcal{C}_{k_{b}}}(\gamma, \theta)$ gives the minimum distance in $\mathcal{S}_{\text {eff }}$. As $\left(\gamma_{i}, \theta\right)$ moves from B to $\mathbf{C}$, $\left|\theta_{b}-\theta\right|$ increases, thus from Lemma 6 , the minimum distance in $\mathcal{S}_{\text {eff }}$, i.e. the value of $d_{\mathcal{C}_{k_{b}}}\left(\gamma_{i}, \theta\right)$ continues to increase. So B can never correspond to the point where the minimum distance in $\mathcal{S}_{\text {eff }}$ is maximized. The proof for the case when $\theta_{i}=0$ is exactly similar to the above proof. This completes the proof.

The procedure to obtain the optimal phase $\theta_{i, o p t}$ of the fade state, for the violation circle centred at singular fade state $\left(\gamma_{i}, \theta_{i}\right) \in \mathcal{H}_{W}$ is stated as follows:

Step 1 Find the points of intersections of the $\operatorname{arc} \gamma=\gamma_{i}, \theta \in$ $[0, \pi / M]$, with the boundaries that satisfy the conditions mentioned in Lemma 7

Step 2 If there is only one such point of intersection, say with the boundary between the regions $\mathcal{R}_{W}\left(\mathcal{C}_{k_{a}}\right)$ and $\mathcal{R}_{W}\left(\mathcal{C}_{k_{b}}\right)$, $\theta_{i, \text { opt }}$ is obtained by solving the equation $d_{\mathcal{C}_{k_{a}}}^{2}(\gamma, \theta)=$ $\left.d_{\mathcal{C}_{k_{b}}}^{2}(\gamma, \theta)\right|_{\gamma=\gamma_{i}}$. On the other hand, if there are $L$ such points of intersections, say with boundaries between regions $\mathcal{R}_{W}\left(\mathcal{C}_{k_{a, l}}\right)$ and $\mathcal{R}_{W}\left(\mathcal{C}_{k_{b, l}}\right), 1 \leq l \leq L$, calculate the phase of each of these points of intersection, $\theta_{l \text {,intersect }}$, $1 \leq l \leq L$ by solving the equation $d_{\mathcal{C}_{k_{a, l}}}^{2}(\gamma, \theta)=$ $\left.d_{\mathcal{C}_{k_{b, l}}}^{2}(\gamma, \theta)\right|_{\gamma=\gamma_{i}}$. Then compute the minimum distances in $\mathcal{S}_{\text {eff }}$ for the fade state corresponding to the point of intersection $\left(\gamma_{i}, \theta_{l \text {,intersect }}\right)$ as $d_{\min }\left(\gamma_{i}, \theta_{l, \text { intersect }}\right)=$ $\left.d_{\mathcal{C}_{k_{a, l}}}^{2}(\gamma, \theta)\right|_{\gamma=\gamma_{i}, \theta=\theta_{l, \text { intersect. }}}$. Choose

$$
l^{\prime}=\arg \max _{1 \leq l \leq L} d_{\min }\left(\gamma_{i}, \theta_{l, \text { intersect }}\right) .
$$

Then we have,

$$
\theta_{i, \text { opt }}=\theta_{l^{\prime}, \text { intersect }} .
$$

The optimal rotation angles for the violation circle with centres at $\left(\gamma_{i}, \theta_{i}\right)$, can now be easily calculated from $\theta_{i, o p t}$. The optimal rotation angle, $\alpha_{i, o p t}$, for the violation circle centred at $\left(\gamma_{i}, \theta_{i}\right)$ is that rotation angle that transforms the fade state from $\left(\gamma_{i}, \theta_{i}\right)$ to $\left(\gamma_{i}, \theta_{i, o p t}\right)$. (See Lemma 5])

For violation circles with centre at $\left(\gamma_{i}, \theta_{i}\right), 1 \leq i \leq N_{W}$, the optimal rotation angles for the User- 2 are as follows:

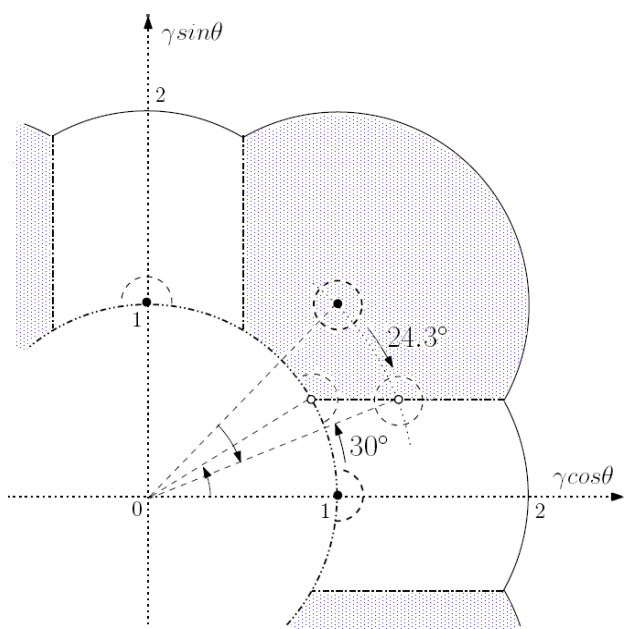

Fig. 9: Optimal rotation angles for the QPSK case

- If $\theta_{i}=\pi / M$, from Lemma [5 the optimal rotation is $\alpha_{i, o p t}=\pi / M-\theta_{i, o p t}$ in a clockwise direction.

- If $\theta_{i}=0$, from Lemma 5, the optimal rotation is $\alpha_{\mathrm{i}, \mathrm{opt}}=$ $\theta_{\mathrm{i}, \text { opt }}$ in an anticlockwise direction.

Example 7: Optimal angles for QPSK signal sets When both users use QPSK constellations at input, the channel quantization is shown in Fig. 3. The optimal rotation angle are calculated as shown below. These are shown in Fig. 9

- For violation circle centred at $\left(\sqrt{2}, \frac{\pi}{4}\right)$, the optimal phase $\theta_{1, \text { opt }}$ is the phase of point of intersection of the arc $\gamma=$ $\sqrt{2}$ and pairwise boundary $d_{\mathcal{C}_{k_{1}}}^{2}(\gamma, \theta)=d_{\mathcal{C}_{k_{2}}}^{2}(\gamma, \theta)$ i.e. $\gamma \sin \theta=0.5$. Therefore, $\theta_{1, \text { opt }}=\sin ^{-1}\left(\frac{1}{2 \sqrt{2}}\right) \approx 20.7^{\circ}$. Thus the optimal rotation angle for the constellation of User-2 relative to User- 1 is $\alpha_{1, o p t}=45^{\circ}-20.7^{\circ}=24.3^{\circ}$ in a clockwise direction.

- For violation circle centred at $(1,0)$, the optimal phase corresponds to the point of intersection of the arc $\gamma=1$ and boundary $d_{\mathcal{C}_{k_{1}}}^{2}(\gamma, \theta)=d_{\mathcal{C}_{k_{2}}}^{2}(\gamma, \theta)$, i.e. $\gamma \sin \theta=0.5$. Thus $\theta_{2, \text { opt }}=\sin ^{-1}(0.5)=30^{\circ}$. Thus the optimal rotation angle for User-2 is $\alpha_{2, \text { opt }}=30^{\circ}$ in an anticlockwise direction relative to User-1.

Example 8: Optimal Angles for 8-PSK signal sets When both users are using 8-PSK constellations at the input, the channel quantization is obtained as shown in Fig. 7 The optimal angles of rotation for User-2 relative to User-1, $\alpha_{i, o p t}$ can be calculated using the same technique for all the violation circles centred at the singular fade states $\left(\gamma_{i}, \theta_{i}\right), 1 \leq i \leq 7$. For each $\left(\gamma_{i}, \theta_{i}\right), 1 \leq i \leq 7$, the boundaries corresponding to the optimal phase, $\theta_{i, o p t}$ and $\alpha_{i, \text { opt }}$ are tabulated in Table IIII. The letters $(\mathrm{C})$ or $(\mathrm{A})$ in the column corresponding to $\alpha_{i, \text { opt }}$ indicates the direction of rotation as clockwise and anticlockwise respectively.

\section{Upper Bound on $\delta$}

In this subsection we obtain an upper bound on $\delta$ for which the proposed rotation scheme can be employed. It is necessary that the violation circle corresponding to any of the singular 
TABLE III: Optimal angles of rotation for 8-PSK case

\begin{tabular}{|l|l|l|l|l|}
\hline$i$ & $\left(\gamma_{i}, \theta_{i}\right)$ & Boundary & $\theta_{i, o p t}$ & $\alpha_{i, o p t}$ (C/A) \\
\hline 1 & $(1,0)$ & $d_{\mathcal{C}_{k_{1}}}^{2}(\gamma, \theta)=d_{\mathcal{C}_{k_{4}}}^{2}(\gamma, \theta)$ & $\tan ^{-1}\left(\frac{1}{2}\right)-\cos ^{-1}\left(\frac{\gamma_{1}^{2}+\frac{1}{2}+\frac{1}{\sqrt{2}}}{\sqrt{5} \gamma_{1}}\right) \approx 17.3^{\circ}$ & 17.3 (A) \\
\hline 2 & $(\sqrt{2}, 0)$ & $d_{\mathcal{C}_{k_{1}}}^{2}(\gamma, \theta)=d_{\mathcal{C}_{k_{5}}}^{2}(\gamma, \theta)$ & $\tan ^{-1}\left(\frac{1}{2 \sqrt{2}-1}\right)-\cos ^{-1}\left(\frac{\gamma_{2}^{2}+2}{2 \gamma_{2} \sqrt{5-2 \sqrt{2}}}\right) \approx 12.4^{\circ}$ & $12.4^{\circ}$ (A) \\
\hline 3 & $(\sqrt{2}+1,0)$ & $d_{\mathcal{C}_{k_{3}}}^{2}(\gamma, \theta)=d_{\mathcal{C}_{k_{7}}}^{2}(\gamma, \theta)$ & $\sin ^{-1}\left(\frac{1}{2 \gamma_{3}}\right) \approx 12^{\circ}$ & $12^{\circ}(\mathrm{A})$ \\
\hline 4 & $\left(\sqrt{4-2 \sqrt{2}}, \frac{\pi}{8}\right)$ & $d_{\mathcal{C}_{k_{1}}}^{2}(\gamma, \theta)=d_{\mathcal{C}_{k_{4}}}^{2}(\gamma, \theta)$ & $\tan ^{-1}\left(\frac{1}{2}\right)-\cos ^{-1}\left(\frac{\left.\gamma_{4}^{2}+\frac{1}{2}+\frac{1}{\sqrt{2}}\right) \approx 15.9^{\circ}}{6.6^{\circ}}\right)$ \\
\hline 5 & $\left(\sqrt{1+\frac{1}{\sqrt{2}}}, \frac{\pi}{8}\right)$ & $d_{\mathcal{C}_{k_{1}}}^{2}(\gamma, \theta)=d_{\mathcal{C}_{k_{5}}}^{2}(\gamma, \theta)$ & $\tan ^{-1}\left(\frac{1}{2 \sqrt{2}-1}\right)-\cos ^{-1}\left(\frac{\gamma_{5}^{2}+2}{2 \gamma_{5} \sqrt{5-2 \sqrt{2}}}\right) \approx 13^{\circ}$ & $9.5^{\circ}(\mathrm{C})$ \\
\hline 6 & $\left(\sqrt{2+\sqrt{2}}, \frac{\pi}{8}\right)$ & $d_{\mathcal{C}_{k_{3}}}^{2}(\gamma, \theta)=d_{\mathcal{C}_{k_{6}}}^{2}(\gamma, \theta)$ & $\cos ^{-1}\left(\frac{1+\sqrt{2}}{2 \gamma_{6}}\right)-\frac{\pi}{4} \approx 4.2^{\circ}$ & $18.3^{\circ}(\mathrm{C})$ \\
\hline 7 & $\left(\sqrt{4+2 \sqrt{2}}, \frac{\pi}{8}\right)$ & $d_{\mathcal{C}_{k_{3}}}^{2}(\gamma, \theta)=d_{\mathcal{C}_{k_{7}}}^{2}(\gamma, \theta)$ & $\sin ^{-1}\left(\frac{1}{2 \gamma_{7}}\right) \approx 11^{\circ}$ & $11.5^{\circ}(\mathrm{C})$ \\
\hline
\end{tabular}

fade states must not overlap with any of the effective shifted circles, otherwise the minimum distance guarantee will be violated. This is illustrated in Fig. 10, It is clear from the figure, to avoid the overlap it is necessary that the distance between the centre of each of the effective shifted circle $\left(\gamma_{i}, \theta_{i, \text { opt }}\right), 1 \leq i \leq N_{W}$ and the singular fade states $\left(\gamma_{j}, \theta_{j}\right)$, $1 \leq j \leq N_{W}$ should be at least equal to sum of the radius of the shifted circle and the radius of the violation circles centred at $\left(\gamma_{j}, \theta_{j}\right)$. It is required, for each $i, 1 \leq i \leq N_{W}$,

$$
\begin{aligned}
\rho\left(\gamma_{i}, \theta_{i}\right)+\rho\left(\gamma_{j}, \theta_{j}\right) & \leq d_{\left(\gamma_{i}, \theta_{i, o p t}\right) \leftrightarrow\left(\gamma_{j}, \theta_{j}\right)} \\
& \text { for all } j, 1 \leq j \leq N_{W},
\end{aligned}
$$

where $d_{\left(\gamma_{i}, \theta_{i, o p t}\right) \leftrightarrow\left(\gamma_{j}, \theta_{j}\right)}$ is the Euclidean distance between the points $\left(\gamma_{j}, \theta_{j}\right)$ and $\left(\gamma_{i}, \theta_{i, \text { opt }}\right)$ in the $(\Gamma, \Theta)$ plane. Since $\rho\left(\gamma_{k}, \theta_{k}\right), 1 \leq k \leq N_{W}$, is a function of $\delta$, (12) provides an upper bound on $\delta$.

Example 9: When both users use QPSK constellations at the input, both the violation circles centred at $\left(1,0^{\circ}\right)$ and $\left(\sqrt{2}, 45^{\circ}\right)$ has radius $\delta / \sqrt{2}$ i.e. $\rho\left(1,0^{\circ}\right)=\rho\left(\sqrt{2}, 45^{\circ}\right)=\frac{\delta}{\sqrt{2}}$. Now,

$$
\begin{aligned}
& d_{\left(\sqrt{2}, 45^{\circ}\right) \leftrightarrow\left(\sqrt{2}, 20.7^{\circ}\right)}=d_{\left(1,0^{\circ}\right) \leftrightarrow\left(\sqrt{2}, 20.7^{\circ}\right)} \approx 0.5936 \\
& d_{\left(\sqrt{2}, 45^{\circ}\right) \leftrightarrow\left(1,30^{\circ}\right)}=d_{\left(1,0^{\circ}\right) \leftrightarrow\left(1,30^{\circ}\right)} \approx 0.5176 .
\end{aligned}
$$

To avoid overlap, from (12), we have

$$
\begin{aligned}
& 2\left(\frac{\delta}{\sqrt{2}}\right) \leq \min \{0.5936,0.5176\} \\
& \therefore \delta \leq 0.365 \approx \delta_{\text {max }} .
\end{aligned}
$$

For $\delta>\delta_{\max }$ there always exist some fade states for which the minimum distance in $\mathcal{S}_{\text {eff }}$ cannot be increased beyond $\delta$ using the proposed scheme. For example, in Fig. 10, the fade state corresponding to the point $P$ is transferred to $P^{\prime}$ after rotation. But $P^{\prime}$ still lies within the violation circle corresponding to singular fade state $\left(\sqrt{2}, \frac{\pi}{4}\right)$, thus minimum distance guarantee is violated.

\section{SIMULATION RESULTS AND DISCUSSIONS}

All through the previous section we have assumed that the ratio $\frac{h_{2}}{h_{1}}$ is calculated at the destination. But even if is not so, i.e. actually the ratio $\frac{h_{1}}{h_{2}}$ is used for channel quantization at the

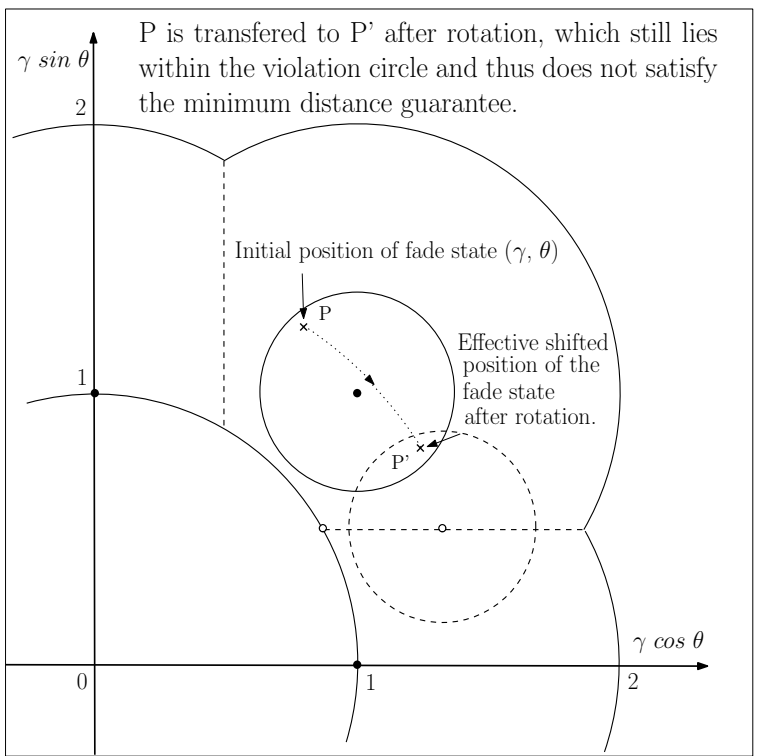

Fig. 10: Diagram illustrating the necessity for an upper bound on $\delta$

destination, then exactly the same scheme would work except that instead of rotating the constellation of User-2 we have to rotate the signal set of User-1. The optimal angles of rotation will still be same as calculated before. The feedback that the destination sends back to the users indicates if the fade state $(\gamma, \theta)$ lies in any of the violation circles or not, and if it does, then identifies in which one of the $N_{W}$ violation circles does it lie in. It also needs to indicate which one among the two ratios is calculated for fade state quantization at the destination. Thus the total feedback overhead is $\left\lceil\log _{2}\left(N_{W}+1\right)\right\rceil+1$ bits. For example, the feedback overhead for the QPSK and 8-PSK case are 3 and 4 bits respectively. This feedback overhead is very nominal.

The system is simulated for the case when both users use QPSK signal sets at the input. The probability of error is plotted against SNR in Fig. 11 both without and with adaptive modulation for different values of $\delta$. The gains obtained increases on increasing $\delta$ as expected. For a $P_{e}=10^{-3}$ there is a $4 \mathrm{~dB}$ gain obtained with adaptive modulation with $\delta=0.35$, 


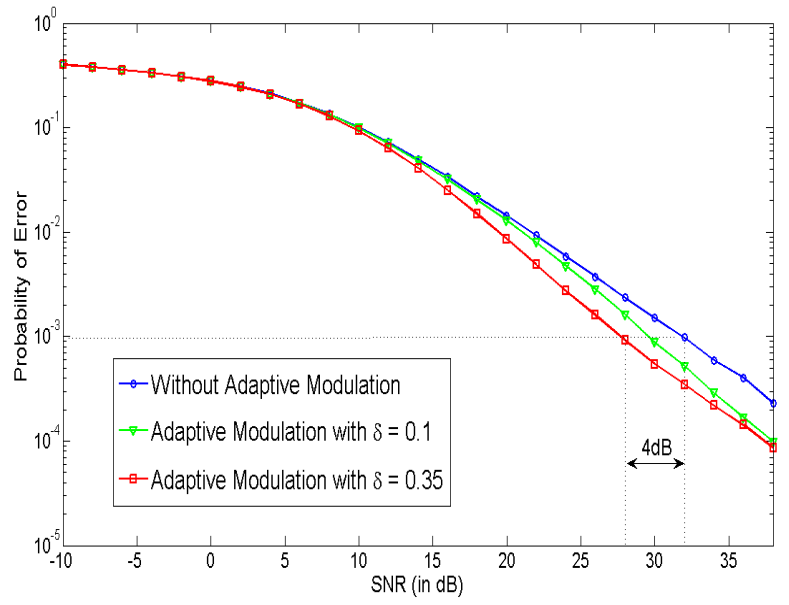

Fig. 11: Probability of error vs SNR plot, when both users use QPSK signal sets

as shown in the figure.

\section{CONCLUSIONS}

In this paper, we have proposed a modulation scheme for the two-user MAC with fading which adapts according to the fade states. For this purpose we have obtained a quantization of all possible fade states based on a minimum distance criteria when both users use $M$-PSK constellations at input. We have identified the regions, called violation circles, such that when the fade state lies in them the minimum distance requirement in $\mathcal{S}_{\text {eff }}$ is violated. The quantized fade state knowledge is fed back to the users using just $\left\lceil\log _{2}\left(N_{W}+1\right)\right\rceil+1$ bits. Based on this quantized feedback, one of the users rotates it's constellation to effectively overcome the 'bad channel conditions'. We have shown the extent to which the error performance of this proposed scheme is better than the conventional scheme without adaptation. The case when both users use QAM constellations at input has not been considered in this paper, is a natural topic for future work. Other cases with more than two users communicating with a single destination is also an interesting direction for future work.

\section{ACKNOWLEDGEMENT}

This work was supported partly by the DRDO-IISc program on Advanced Research in Mathematical Engineering through a research grant as well as the INAE Chair Professorship grant to B. S. Rajan.

\section{REFERENCES}

[1] Thomas M. Cover and Joy A. Thomas Elements of Information Theory,Second Edition, New York: Wiley 1999

[2] R. Gallager, "A perspective on multiaccess channels", IEEE Trans. on Inf. Theory, Vol. 31, No. 2, pp. 124-142, Mar. 1985

[3] J. Harshan and B. Sundar Rajan, "On Two-User Gaussian Multiple Access Channels with Finite Input Constellations ", IEEE Trans.on Inf. Theory, Vol.57, No. 3,pp. 1299-1327,Mar. 2011

[4] J. Harshan and B. Sundar Rajan, "A Constellation Power Allocation Scheme for Two-User Gaussian MAC ", Proc of IEEE PIMRC'11

[5] T. Koike-Akino, P. Popovski and V. Tarokh, "Optimized constellation for two-way wireless relaying with physical network coding", IEEE Journal on selected Areas in Comm., Vol.27, pp. 773- - 787, June 2009.
[6] Vijayvaradharaj T Muralidharan, Vishnu Namboodiri and B. Sundar Rajan, "Channel Quantization for Physical-Layer Network Coded Two-Way Relaying", available online at arXiv:1109.6101,1 [cs.IT], 28 Sept.2011.

[7] Haghi, A. Khosravi-Farsani, R. Aref, M. R. Marvasti, "The capacity region of fading Multiple Access Channels with cooperative encoders and partial CSIT",Proc. of IEEE ISIT,2010, pp.485-489, June 2010

[8] Y Cemal, Y. Steinberg "The Multiple Access Channel with Partial State Information at the Encoders" ,IEEE Trans. on Inf Theory Vol 51, No.11, pp. 3392-4003,2005

[9] Moritz Wiese, Holger Boche and Igor Bjelakovic "The compound MAC with common message and partial channel state information",IEEE ISITA 2010, Vol 1, pp. 416-421 
This figure "optimize.PNG" is available in "PNG" format from: http://arxiv.org/ps/1201.3088v1 